Novel Tools and Methods

\title{
General Anesthesia Disrupts Complex Cortical Dynamics in Response to Intracranial Electrical Stimulation in Rats
}

\author{
(i)A. Arena, ${ }^{1}$ R. Comolatti, ${ }^{2}$ S. Thon, ${ }^{1}$ A. G. Casali, ${ }^{2}$ and (DJ. F. Storm ${ }^{1}$
}

https://doi.org/10.1523/ENEURO.0343-20.2021

${ }^{1}$ Department of Molecular Medicine, University of Oslo, Oslo 0372, Norway and ${ }^{2}$ Institute of Science and Technology, Federal University of São Paulo, São José dos Campos, 12247-014, Brazil

\section{Visual Abstract}
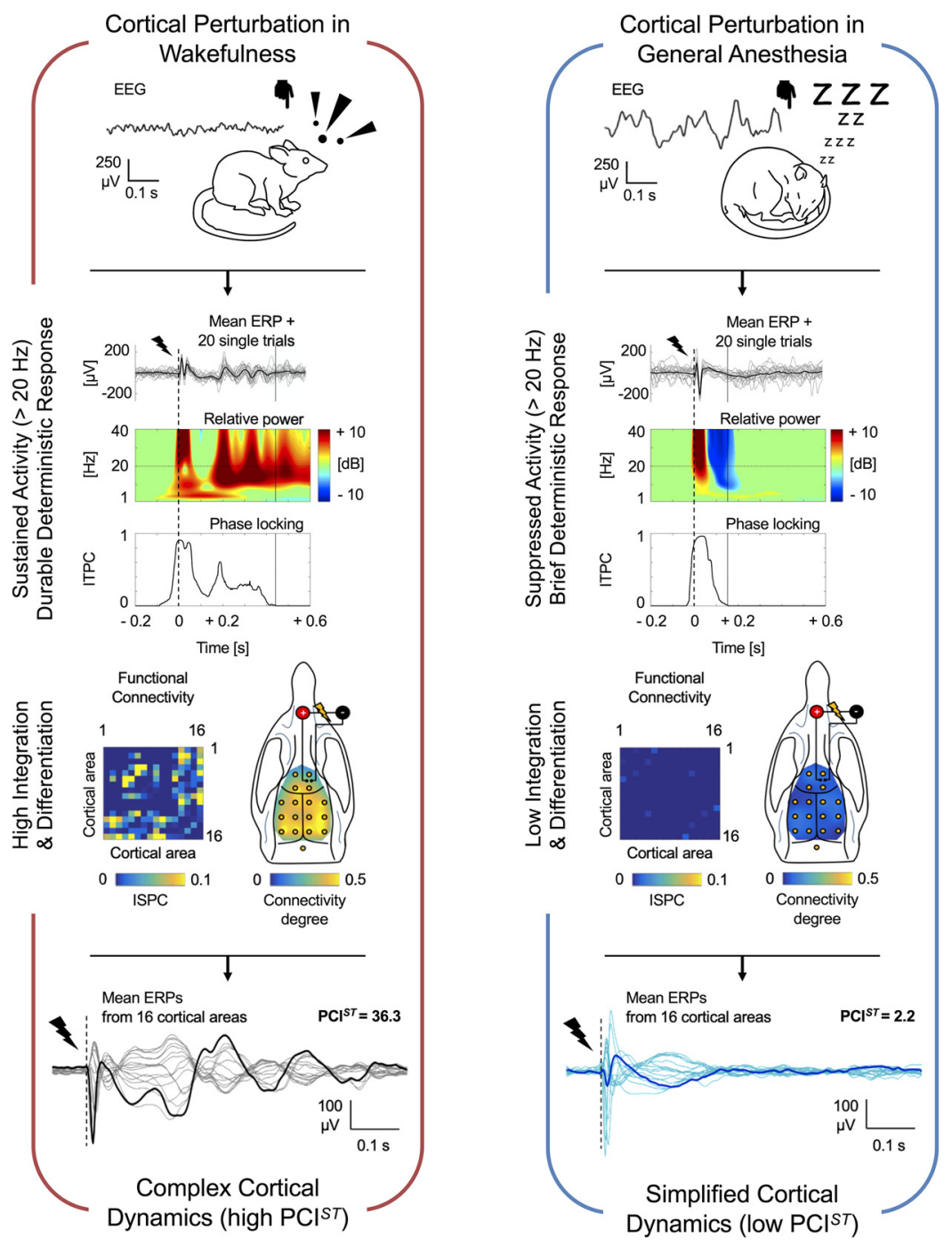


\section{Significance Statement}

The perturbational complexity index $(\mathrm{PCl})$ is an electrophysiological metric for the capacity of cortical circuits to integrate information. $\mathrm{PCl}$ proved to be a reliable, objective, report-independent index that discriminates between conscious and unconscious states in humans, with promising clinical implications in brain disorders. However, the neural mechanisms underlying PCI remain uncertain and difficult to test, because the method has never been directly applied to non-human species in vivo before. Here, we reproduce $\mathrm{PCl}$ in rats, thus setting the stage for invasive, mechanistic investigations. We show how PCl correlates with functional connectivity and pattern diversity, and collapses from wakefulness to general anesthesia. Finally, we provide evidence for the role of transient, sleep-like putative periods of neuronal silence in preventing complex cortical interactions.

The capacity of human brain to sustain complex cortical dynamics appears to be strongly associated with conscious experience and consistently drops when consciousness fades. For example, several recent studies in humans found a remarkable reduction of the spatiotemporal complexity of cortical responses to local stimulation during dreamless sleep, general anesthesia, and coma. However, this perturbational complexity has never been directly estimated in non-human animals in vivo previously, and the mechanisms that prevent neocortical neurons to engage in complex interactions are still unclear. Here, we quantify the complexity of electroencephalographic (EEG) responses to intracranial electrical stimulation in rats, comparing wakefulness to propofol, sevoflurane, and ketamine anesthesia. The evoked activity changed from highly complex in wakefulness to far simpler with propofol and sevoflurane. The reduced complexity was associated with a suppression of high frequencies that preceded a reduced phase-locking, and disruption of functional connectivity and pattern diversity. We then showed how these parameters dissociate with ketamine and depend on intensity and site of stimulation. Our results support the idea that brief periods of activity-dependent neuronal silence can interrupt complex interactions in neocortical circuits, and open the way for further mechanistic investigations of the neuronal basis for consciousness and loss of consciousness across species.

Key words: anesthesia; connectivity; consciousness; OFF period; perturbational complexity index; rat

\section{Introduction}

A longstanding challenge in neuroscience has been the identification of robust and neuron-based measures of consciousness. Recently, brain complexity, defined as the combination of functional differentiation and

Received August 10, 2020; accepted June 3, 2021; First published July 22, 2021.

The authors declare no competing financial interests.

Author contributions: A.A. and J.F.S. designed research; A.A. and S.T. performed research; R.C. and A.G.C. contributed unpublished reagents/ analytic tools; A.A., R.C., S.T., and A.G.C. analyzed data; A.A., R.C., A.G.C., and J.F.S. wrote the paper.

This work was supported by the European Union's Horizon 2020 Framework Programme for Research and Innovation under the Specific Grant Agreements Nos. 785907 (Human Brain Project SGA2) and 720270 (Human Brain Project SGA1; to J.F.S.) and by São Paulo Research Foundation (FAPESP) Grants 2016/08263-9 (to A.G.C.) and 2017/03678-9 (to R.C.).

R. Comolatti's present address: Department of Biomedical and Clinical Sciences "L. Sacco," University of Milan, Milan 20157, Italy.

Acknowledgements: We thank Per M. Knutsen for help in preparation of the experimental setup and Charlotte Boccara for advice regarding the writing. We also thank Marcello Massimini, Andrea Pigorini, Matteo Fecchio, and Simone Russo for their valuable comments, suggestions, and support along the way.

Correspondence should be addressed to A. Arena at alessandro.arena@ medisin.uio.no or J. F. Storm at j.f.storm@medisin.uio.no.

https://doi.org/10.1523/ENEURO.0343-20.2021 Copyright @ 2021 Arena et al.

This is an open-access article distributed under the terms of the Creative Commons Attribution 4.0 International license, which permits unrestricted use, distribution and reproduction in any medium provided that the original work is properly attributed. integration in thalamocortical systems, has gained growing attention as promising candidate (Casali et al., 2013; Dehaene, 2014; Koch et al., 2016). For example, a reliable association between conscious states and complexity of global network dynamics has been demonstrated by means of both electroencephalography (EEG; Schartner et al., 2015) and imaging of spontaneous brain activity (Demertzi et al., 2019). A highly accurate method to assess complexity of causal, cortical dynamics is the perturbational complexity index $(\mathrm{PCl})$, which was originally introduced and validated for discrimination between unconscious and conscious unresponsive patients (Casali et al., 2013; Casarotto et al., 2016). PCl is based on a perturbational approach: brief transcranial magnetic stimulation (TMS) or intracranial electrical stimulation are used to trigger cortical activity, and the spatiotemporal complexity of the EEG-recorded event-related potentials (ERPs) is quantified. Long-lasting responses that are both temporally differentiated and distributed among cortical areas (high $\mathrm{PCl}$ ) are evoked whenever subjects have conscious experiences, such as during wakefulness, rapid eye movement (REM) sleep or ketamine anesthesia, when dreams or hallucinations occur (Casali et al., 2013; Sarasso et al., 2015; Casarotto et al., 2016; Rosanova et al., 2018; Comolatti et al., 2019). In contrast, during dreamless non-REM (NREM) sleep, general anesthesia, and unresponsive wakefulness syndrome (UWS; or "vegetative state"), the ERPs appear to be 
either local and short-lasting (less integrated), or global and stereotyped (less differentiated), yielding low $\mathrm{PCl}$ (Casali et al., 2013; Sarasso et al., 2015; Casarotto et al., 2016; Rosanova et al., 2018; Comolatti et al., 2019).

Although the PCl method has been thoroughly tested in humans, it has previously never been directly transferred to any non-human species in vivo. Therefore, it is not clear to what extent rodent brains can sustain the complex dynamics in response to brief direct stimulation that are characteristic of conscious brain states in humans. Developing animal models and extending the application of $\mathrm{PCl}$ is of paramount importance, since the neuronal mechanisms underlying the engagement or interruption of complex cortical activations are still uncertain, and mechanistic investigations may lead to intervention strategies for restoring complexity and awareness in brain-injured, unconscious patients. Here, we implement and test the $\mathrm{PCl}$ method in rats. By measuring the complexity of intracranial EEG responses to cortical stimulation, we show that the awake rat brain supports complex cortical activations, which turn into simplified, less integrated responses during general anesthesia. We further demonstrate that the disruption of long-lasting complex activations is associated with suppression of high-frequency (HF) EEG power and reduced phase-locking, supporting the hypothesis that neuronal hyperpolarization might prevent cortical neurons from engaging in durable, complex interactions (Pigorini et al., 2015; Tononi et al., 2016; Sanchez-Vives et al., 2017; Storm et al., 2017). The main results of this study have previously been presented in abstract form at conferences and as a preprint (Arena et al., 2018, 2020).

\section{Materials and Methods}

\section{Animal model}

Experimental and animal care procedures were conducted at the University of Oslo and were approved by the Norwegian Authority Mattilsynet (FOTS ID: 11812) in agreement with Norwegian law of animal handling. Experiments were conducted on adult male Sprague Dawley rats $(\sim 300-500 \mathrm{~g}, n=12)$. All efforts have been made to avoid/minimize animals' distress and pain during the entire course of experimentation. Rats were caged in enriched environments, with ad libitum access to food and water and were exposed to $12 / 12 \mathrm{~h}$ light/dark cycle at $23^{\circ} \mathrm{C}$ constant room temperature. The experiments were conducted during the afternoon within the light phase of the cycle.

\section{Electrodes and surgical procedure}

All coordinates for electrodes implantation are expressed referring to bregma position, $\mathrm{x}=$ medial-lateral axis (-, left hemisphere; +, right hemisphere), $y=$ rostralcaudal axis (-, caudal to bregma; +, rostral to bregma), $\mathrm{z}=$ dorsal-ventral axis. ERPs were triggered by electrical stimulations delivered by a bipolar electrode chronically implanted in right secondary motor cortex (M2), composed by two insulated tungsten wires $(50-\mu \mathrm{m}$ caliber; $\sim 500-\mu \mathrm{m}$ distance between wires), and recorded by 16 stainless steel screws (1.2-mm caliber) electrodes. The bipolar electrode was inserted perpendicularly to the cortical surface, along the coronal plane and the coordinates (in $\mathrm{mm}$ ) for implantation were: $\mathrm{x}=+1.2$ left wire/ +1.7 right wire; $y=+3.7 ; z=+1.9$. Recording electrodes were in contact with the dura and were organized in a grid, symmetric along the sagittal suture, and were placed at the following coordinates (in mm): $x= \pm 1.5, y=+5$ (M2); $x=$ $\pm 1.5, y=+2(\mathrm{M} 2) ; \mathrm{x}= \pm 1.5, \mathrm{y}=-1$ (primary motor cortex; $\mathrm{M} 1$ ); $\mathrm{x}= \pm 4.5, y=-1$ (primary somatosensory cortex; $\mathrm{S} 1$ ); $\mathrm{x}= \pm 1.5, \mathrm{y}=-4$ (retrosplenial cortex; RS); $\mathrm{x}= \pm 4.5, \mathrm{y}=-4$ (parietal associative cortex; PA); $x= \pm 1.5, y=-7$ (secondary visual cortex; V2); $\mathrm{x}= \pm 4.5, \mathrm{y}=-7$ (primary visual cortex; $\mathrm{V} 1$ ); $\mathrm{x}=0, \mathrm{y}=-10$ (cerebellum, ground; GND). Impedances of all electrodes were measured in situ at $1 \mathrm{kHz}$ at the beginning of each recording session, for each rat. The averaged impedance across all sessions, channels and animals was $0.17 \pm 0.03 \mathrm{M} \Omega$ for stimulating electrodes and $7.12 \pm 0.42 \mathrm{k} \Omega$ for recording electrodes (mean \pm SEM).

Surgical implantation of electrodes was performed under controlled, deep anesthesia with sevoflurane 2.5$5 \%$ (Baxter) in oxygen concentrated $\left(\mathrm{O}_{2}>85 \%\right.$ ), humidified room air (constant rate: $0.5 \mathrm{l} / \mathrm{min}$ ). Body temperature was maintained at $36.5-37.5^{\circ} \mathrm{C}$ by a heating pad, and subcutaneous injections of butorphanol $(2 \mathrm{mg} / \mathrm{kg})$ and dexamethasone $(0.2 \mathrm{mg} / \mathrm{kg})$ were given. Absence of response to painful stimulation was ensured and standard sterile procedures were used throughout the surgical operation. Holes were drilled through the exposed skull at the desired coordinates, with stereotaxic guidance. Electrodes were positioned and two machine screws were also upside-down mounted over the caudal part of the skull for subsequent head restriction. Dental acrylic was applied over the entire exposed skull, sealing the wound margins and securing electrodes in place. After surgery, buprenorphine $(0.1 \mathrm{mg} / \mathrm{kg})$ and meloxicam $(1 \mathrm{mg} / \mathrm{kg}$ ) were subcutaneously injected. For the following 3- to 4-d rats were checked for possible signs of distress, infection or damages to electrode implantation. Buprenorphine $(0.1-0.05 \mathrm{mg} / \mathrm{kg})$ and dexamethasone $(0.2-0.1 \mathrm{mg} / \mathrm{kg})$ were also subcutaneously injected once a day.

\section{Experimental procedures}

After recovery, rats were gradually habituated in three consecutive days to body and head restriction in a custom-made recording setup, consisting of a horizontally oriented, elevated transparent acrylic cylinder, in which the rat was introduced (with only the head and the tail outside). Right above the tube, two clamps fixed a head-bar that was connected to the machine screws that were chronically implanted on the skull of the rat, over the cerebellum. The head-bar was adjusted and clumped to sustain and fix the head of the rat in a natural and stable position so that no objects interfered with the natural whisking behavior during wakefulness. A heating blanket also wrapped the bottom half of the acrylic tube to keep the body temperature of the rat at $36.5-37.5^{\circ} \mathrm{C}$ during general anesthesia. After one week from surgery, rats 
underwent several electrophysiological stimulation/recording sessions during wakefulness, followed by general anesthesia. Recording sessions were interleaved with a resting period of at least $48 \mathrm{~h}$. Three different general anesthetics were tested (sevoflurane, propofol and ketamine) in randomized different days and most of the rats were exposed to all anesthetics. At the end of the last recording session, during general anesthesia, an electrolytic lesion was performed by applying $30 \mu \mathrm{A}$ for $30 \mathrm{~s}$ to the poles of the stimulating electrode. Rats were then killed with a lethal dose of pentobarbital $(140 \mathrm{mg} / \mathrm{kg}$, i.v.) and, after the suppression of corneal reflex, intracardially perfused with PBS (phosphate buffer solution with heparin $5000 \mathrm{IU} / \mathrm{I}$ ) and $4 \%$ paraformaldehyde in PBS at $4^{\circ} \mathrm{C}$ for tissue fixation. Brains were then extracted and processed for histologic Nissl staining.

During the recording session the tail vein was cannulated (26-G catheter) for allowing intravenous infusion of propofol or ketamine, when needed and the stimulating electrode was connected to an isolated current stimulator (Isolator HG203, High Medical) triggered by a voltage pulse generator (2100, AM Systems). Recording electrodes were connected to a 16-channel unipolar amplifier board with common reference shorted to ground (RHD2132, Intan Technologies), controlled by Open Ephys system (Siegle et al., 2017), and the epidural EEG signal was acquired and digitized at 10 or $30 \mathrm{kHz}, 16$-bit resolution. Stimulation/recording was conducted in darkness after $\sim 45$ min of acclimatization in the setup. Depending on the randomized recording session, rats were exposed to several electrical monophasic current pulses (duration $1 \mathrm{~ms}$ ) of $50 \mu \mathrm{A}$ or of various intensities $(40,60,80$, and $100 \mu \mathrm{A}$, organized in randomized blocks) delivered at $0.1 \mathrm{~Hz}$ during wakefulness. Stimulations were repeated during subsequent general anesthesia within the same animal. General anesthesia was induced either by exposure to sevoflurane $5 \%$, or by the intravenous bolus injection of propofol $10 \mathrm{mg} / \mathrm{kg}$ (B-Braun) or ketamine $30 \mathrm{mg} / \mathrm{kg}$ (Vetoquinol), then maintained at the initial constant dosage of either sevoflurane $2.5 \%$ (by a gas mask, in humidified air, $\mathrm{O}_{2}>85 \%$, at $0.5 \mathrm{I} / \mathrm{min}$ ), propofol $1 \mathrm{mg} / \mathrm{kg} / \mathrm{min}$ or ketamine $1.75 \mathrm{mg} /$ $\mathrm{kg} / \mathrm{min}$ (by a syringe pump). Subcutaneous injection of glycopyrrolate $0.01 \mathrm{mg} / \mathrm{kg}$ was also performed during ketamine anesthesia to reduce the induced increase in salivation. Eye ointment was applied to keep eyes moist and body temperature was kept at $36.5-37.5^{\circ} \mathrm{C}$ by a heating blanket system. The initial dosages were chosen in accordance with the literature (Idvall et al., 1980; Brammer et al., 1993; Benito et al., 2010; Arena et al., 2017) and adjusted in a set of pilot experiments on five rats, in which the loss of righting reflex was evaluated by gently laying down the rats on their backs, on a flat surface, without any physical constraint. Any muscular movements toward acquiring a normal, upright position were considered as a sign of reflex maintenance. The final initial dosages were the minimal for maintaining loss of righting reflex in $100 \%$ of the tested animals in the pilot experiments. At these dosages, reactions to pain stimulations were still present. Thus, for the experiments presented here, all rats were checked for reaction to pain stimulations (pinching the tail by a forceps) after $10 \mathrm{~min}$ from induction, and the concentration (for sevoflurane) or infusion rates (for propofol and ketamine) were increased in a stepwise manner, in steps of $4 \%$ of the initial dosage with $3 \mathrm{~min}$ between each increment, until the pain response was absent. The resulting averaged experimental dosages across rats and sessions were: sevoflurane $2.58 \pm 0.03 \%$, propofol $1.06 \pm 0.02 \mathrm{mg} / \mathrm{kg} / \mathrm{min}$ and ketamine $1.83 \pm 0.03 \mathrm{mg} / \mathrm{kg} / \mathrm{min}$ (mean $\pm \mathrm{SEM}$ ). We did not detect any spontaneous body movements during general anesthesia, by visual inspection through the wall of the transparent acrylic tube used to maintain the rat in position.

A subset of animals was exposed to a final stimulation/recording session with the purpose to detect putative movements of whiskers induced by electrical stimulation of M2. Whiskers of the left mystacial pad were clipped, except for the most caudal vibrissa of the third row (C1), which was tracked by a high-speed camera (500 frame/s, Blackfly S. Mono 0.4 MP USB3, FLIR System). Experimental area was constantly illuminated only by dim red light (wavelength $655 \mathrm{~nm}$, LED, Quadica), which is not detectable by rat retina (Jacobs et al., 2001; De Farias Rocha et al., 2016). A second LED $655 \mathrm{~nm}$ was triggered by the stimulation system, signaling the onset of current pulses to the camera. During wakefulness, head and body restrained rats were exposed to several monophasic current pulses of $1 \mathrm{~ms}$ at either 50 or 100 $\mu \mathrm{A}$, or to train stimulations as positive control (Brecht et al., 2004; train duration $0.3 \mathrm{~s}$, composed by 11 single monophasic current pulses of $1 \mathrm{~ms}$ and $50 \mu \mathrm{A}$, rate of pulse $33 \mathrm{~Hz}$ ). Different stimulations were grouped in randomized blocks and delivered at a rate of $0.2 \mathrm{~Hz}$. In order to avoid saturation artefacts in the amplification system during train stimulation, the epidural EEG activity was recorded by a bipolar amplifier board (RHD2216, Intan Technologies) controlled by Open Ephys system (Siegle et al., 2017), and the frontal-occipital (M2-V2) derivation, ipsilateral to the stimulation, was adopted for only this session. At the end of stimulation/recording, rats were deeply anaesthetized (sevoflurane 5\%) and killed with a lethal dose of pentobarbital $(140 \mathrm{mg} / \mathrm{kg}$, i.v).

\section{Analysis of electrophysiological signal}

Analysis of electrophysiological data were performed in MATLAB2016a (The MathWorks) and Origin 9.1 (OriginLab). Raw epidural EEG recordings were visually inspected to remove channels containing noise artefacts or having impedance $>1 \mathrm{M} \Omega$. EEG signals from all electrodes were re-referenced to the common average across channels for analyzing ERPs, while a bipolar frontal-occipital derivation (M2-V2 right) was chosen for analyzing spontaneous activity. Stimulus artefacts were removed and signal was spline interpolated in a time window from 0 to $0.005 \mathrm{~s}$ from stimulus onset. EEG signal was band pass filtered from 0.5 to $80 \mathrm{~Hz}$ (Butterworth filter, 3rd order) and down-sampled to $500 \mathrm{~Hz}$. ERP epochs from -5 to $5 \mathrm{~s}$ centered at the stimulus onset $(0 \mathrm{~s})$ were then extracted for each channel. All epochs were offset corrected by subtracting the average voltage 
of their respective baseline (from -1 to $0 \mathrm{~s}$ ). Trials with high voltage artefacts in their baseline were removed. Threshold for rejection was set at the averaged root mean square (rms) of baseline (from -1 to $0 \mathrm{~s}$ ) across trials $+3 \mathrm{SDs}$. The first $n=90$ consecutive trials of preprocessed signal were used for analysis of evoked responses to electrical perturbations of $50 \mu \mathrm{A}$ and spontaneous EEG activity was quantified from baseline epochs of $4 \mathrm{~s}$ (from -5 to $-1 \mathrm{~s}$ ) from the corresponding trials (with the exception of one animal in one wakefulness condition, for which 80 trials were used in analysis instead). Whereas $n=56$ consecutive ERPs were used for analysis of evoked responses to current simulations of increasing intensities.

In order to quantify the different states of brain activity induced by general anesthetics in relation to wakefulness, fast Fourier transform (FFT) was performed on $n=90$ epochs of spontaneous EEG activity and normalized by the number of samples $\mathrm{N}$. The squared modules of the normalized FFTs were computed and the resulting power spectrums were averaged across trials. The $1 / f$ relation of the averaged periodogram was then linearly fitted in LogLog coordinates, in the frequency range from 20 to $40 \mathrm{~Hz}$. The slope of the obtained linear function was considered to be the spectral exponent of the $1 / f$ function and was used to quantify the (re)distribution of frequency powers in the spontaneous EEG activity (Colombo et al., 2019).

The amplitude of the evoked response was quantified by the rms amplitude of the first deflection of the mean ERP (0.006-0.05 s from stimulus onset) from each electrode and then averaged across channels.

The slow component (SC) of the ERP was quantified from low-pass filtered responses $(<4 \mathrm{~Hz}$, Butterworth filter, 3rd order). To test any dependency on the spontaneous slow activity, we computed the rms amplitude of the spontaneous SC in a long baseline period (-2-0 s) for each single trial and then averaged across trials and channels, and compared with the rms amplitude of the evoked SC (0-0.3 s), also computed for each single trial, then averaged across trials and channels. The maximal amplitude and latency of the evoked SC were obtained for each channel, from the maximal absolute peak of the ensemble average across trials (SC max), within the time range 0-0.6 s.

Morlet wavelet convolution was performed on each trial for all channels to extract both spectral powers and phases of ERPs. A total of 40 wavelets (three cycles) linearly spanning from 1 to $40 \mathrm{~Hz}$ were adopted. Powers from each channel, trial and frequency were normalized over the averaged power across trials in baseline window $(-0.5$ to $-0.2 \mathrm{~s})$ for each respective frequency and channel. Relative powers were then averaged across trials for each channel and frequency, and converted in $\mathrm{dB}$. To identify only the significant positive and negative variations of $\mathrm{dB}$ with respect to baseline, bootstrap statistic was performed (500 permutations; positive and negative thresholds, $\alpha=0.05$ ) and not significant values were set to 0 . The spectral content of the ERP was quantified by averaging the relative powers in frequency bands: $\delta$ (1$4 \mathrm{~Hz}), \theta(5-7 \mathrm{~Hz}), \alpha(8-14 \mathrm{~Hz}), \beta(15-25 \mathrm{~Hz})$, and $\gamma(26-$ $40 \mathrm{~Hz}$ ), then across time (from 0 to $0.5 \mathrm{~s}$ ) and across channels. The putative "OFF periods" were detected as power suppression $(<0 \mathrm{~dB})$ in the HF range $(20-40 \mathrm{~Hz})$ after stimulation (Pigorini et al., 2015; Rosanova et al., 2018). Starting and end time points of periods of HF suppression were identified in a time window from 0 to $0.3 \mathrm{~s}$ as first downward and last upward zero crossing of the resulting $\mathrm{dB}$ signal. Minimum $\mathrm{dB}$ peak in the same time window was detected, and the HF power was quantified by averaging the relative power in the frequency range of $20-40 \mathrm{~Hz}$, from 0.08 to $0.18 \mathrm{~s}$. The starting point of the time window used to quantify HF power was empirically determined and was the mean starting point of HF suppression across anesthetic conditions. Potential later increments of HF power $(>0 \mathrm{~dB})$ were detected in a time window from 0.08 to $0.8 \mathrm{~s}$. We also quantified increments of low-frequency (LF) activity by averaging the relative power in range $1-4 \mathrm{~Hz}$ and by detecting the maximal positive $\mathrm{dB}$ peak in time range $0-0.6 \mathrm{~s}$.

The deterministic effect that the electrical stimulation had on the EEG response was measured as the duration of increased phase-locking of subsequent ERPs compared with baseline (Pigorini et al., 2015; Rosanova et al., 2018). Phaselocking at each channel, frequency and time point was computed across trials as intertrial phase clustering (ITPC; Cohen, 2014), with the following formula:

$$
I_{T P C}=\left|n^{-1} \sum_{r=1}^{n} e^{i k_{t r t}}\right| .
$$

Where $n$ is the number of trials and $e^{i k}$ is the complex polar representation of the phase $k$ from the trial $r$ at timefrequency point $t f$. ITPC can assume values from 0 (no phase-locking) to 1 (maximal phase-locking), and bootstrap statistic (500 permutations; threshold, $\alpha=0.01$ ) was performed for each frequency and channel, to conserve only the statistically significant increments of ITPC with respect to baseline (from -0.5 to $-0.2 \mathrm{~s}$ ). Significant ITPC values were averaged across a broad band frequency range $(8-40 \mathrm{~Hz}$; Pigorini et al., 2015; Rosanova et al., 2018), and the "ITPC drop" was defined as the time point of the last significant mean ITPC value in time window 0 $0.8 \mathrm{~s}$. The latency of the maximal ITPC value in LF range (1-4 Hz, LF ITPC max) was also computed for each channel and then averaged.

A similar phase-based approach was adopted to quantify increments in functional connectivity across channels following the stimulation compared with baseline. For each trial, phase differences across channels at each frequency and time point were computed and the clustering over trials of resulting phase differences was defined as intersite phase clustering (ISPC; Cohen, 2014) and calculated with the following formula:

$$
I S P C_{t f}=\left|n^{-1} \sum_{r=1}^{n} e^{i\left(k_{x}-k_{y}\right) t r}\right| .
$$

Where $n$ is the number of trials and $e^{i\left(k_{x}-k_{y}\right)}$ is the complex polar representation of the difference between the phases $k_{x}$ and $k_{y}$ from the channels $x$ and $y$, for the trial $r$ at time-frequency point $t f$. Therefore, ISPC represents the consistency of the phase configuration between the 
activity from two channels across trials, at each time-frequency point. ISPC of each channel pair and frequency is then baseline corrected by subtracting the corresponding average value in the time window from -0.5 to $-0.2 \mathrm{~s}$ and bootstrap statistic (500 permutations; positive and negative thresholds, $\alpha=0.05$ ) was performed to maintain only the statistically significant ISPC variations from baseline. All ISPC scores that could be explained by volume conduction (clustering of phase difference around 0 or $\pi$ ) were excluded from the analysis and the resulting ISPC values from each channel pair were averaged in the frequency range $5-14 \mathrm{~Hz}$ in two time windows, during the period of HF suppression (0.08-0.18 s) and immediately later $(0.18-0.3 \mathrm{~s})$. The ISPC scores $>0$ represented significant functional connections between channel pairs and the connectivity degree (CD) was defined as the number of significant functional connections for each electrode, normalized over the number of channels minus one (to exclude autocorrelation). Rats with more than two removed channels (broken or noisy electrodes) were excluded from the connectivity analysis.

The spatiotemporal complexity of EEG responses to electrical stimulations was calculated using the recently introduced $\mathrm{PCl}$ state-transition $\left(\mathrm{PCl}^{\mathrm{ST}}\right.$; Comolatti et al., 2019), which quantifies the number of state transitions (NST) present in the principal components of the signal's response. Briefly, the principal components accounting for $99 \%$ of the variance present in the response are obtained through singular value decomposition and then selected based on a minimum signal-to-noise ratio $\left(\mathrm{SNR}_{\mathrm{min}}\right)$. Then, for each component the number of significant state transition ( $\triangle \mathrm{NST}$ ), a metric derived from recurrent quantification analysis (Marwan et al., 2007), is computed. $\mathrm{PCl}^{S T}$ is the product between the number of principal components surviving dimensionality reduction and the average NST across components (Comolatti et al., 2019). Hence, $\mathrm{PCl}^{S T}$ is high when a response displays multiple, linearly independent components (spatial differentiation), each contributing with significant amounts of state transitions (temporal complexity). In order to minimize the amount of baseline-like oscillations (noise) that contributed to the $\mathrm{PCl}^{\mathrm{ST}}$ value, $\mathrm{SNR}_{\min }$ was chosen using a bootstrap procedure in the following way: for each signal, $\mathrm{PCl}^{\mathrm{ST}}$ was calculated on 16 surrogates for which complexity should be zero, generated using two random non-response $0.5 \mathrm{~s}$ segments ( $t<0 \mathrm{~s}$ or $t>1.5 \mathrm{~s}$, where $0 \mathrm{~s}$ is the stimulation onset) as baseline and response. $\mathrm{SNR}_{\min }$ was then set to 1.8 for all analyses, the smallest value for which the median $\mathrm{PCl}^{\mathrm{ST}}$ across all surrogates was zero. The baseline and response window were defined as from -0.5 to $-0.005 \mathrm{~s}$ and from 0 to $0.6 \mathrm{~s}$ form the stimulus onset, respectively, in accordance with previous experiments with intracranial stimulation in humans (Comolatti et al., 2019). To assess how the complexity of the EEG responses varied in time, $\mathrm{PCl}^{\mathrm{ST}}$ was calculated in shorter $0.1 \mathrm{~s}$ sliding windows from stimulus onset $(0.02-\mathrm{s}$ shift, until $1.1 \mathrm{~s})$ and in the time range $0.08-0.6 \mathrm{~s}$ from the stimulus onset. $\mathrm{PCl}^{S T}$ was computed using the available code at https://github. com/renzocom/PClst and further parameters were set as previously reported (Comolatti et al., 2019).

\section{Analysis of whisker tracking}

The video recording of the whisker movements in response to electrical stimulation was initially analyzed in Bonsai software (Lopes et al., 2015). The centroid of the whisker was tracked offline, and the relative space coordinates in the Cartesian plane were extracted for each frame and imported in MATLAB. Whisker positions were converted into degrees in the polar plane, obtaining angular oscillations in time that were analyzed similarly to the voltage signal. From the continuous signal, $n=21$ consecutive motor responses centered on the stimulus onset $(0$ s) were extracted (from -2.5 to $2.5 \mathrm{~s}$ ). Each motor trial was offset corrected by subtracting the respective mean angle of the baseline (time window from -1 to $0 \mathrm{~s}$ ) and all the analyses were performed at the level of single trials for each rat. The magnitude of the whisker oscillation in response to the stimulus was quantified by the rms amplitude in a time window of $0.25 \mathrm{~s}$ following the stimulus offset and compared with the rms of the baseline (from -0.5 to $-0.25 \mathrm{~s})$. The mean spectral power of the whisker oscillation was also computed. A three-cycle Morlet wavelet convolution was performed with a family of 100 wavelets spanning linearly from 1 to $100 \mathrm{~Hz}$. The powers of each frequency from all trials were extracted and normalized over the corresponding mean power across trials in the baseline (from -0.8 to $-0.3 \mathrm{~s}$ ). The relative powers of each frequency were then averaged over trials and converted into $\mathrm{dB}$. Bootstrap statistic (500 trial-based permutations, thresholds $\alpha=0.05$ ) was performed and the nonsignificant angle variations with respect to the baseline were set to 0 . The resulting relative powers were then averaged in a broad band frequency range (from 5 to $100 \mathrm{~Hz}$ ), in the first $0.25 \mathrm{~s}$ after the stimulus offset.

\section{Histologic staining}

After fixation, brains were exposed to increasing concentrations of sucrose $(10 \%, 20 \%, 30 \%)$ in PBS solutions at $4^{\circ} \mathrm{C}$ for $4 \mathrm{~d}$. Brains were quickly frozen in sucrose $30 \%$ in PBS and sliced in coronal sections of $50-\mu \mathrm{m}$ thickness with a sliding microtome. Coronal sections were then prepared for Nissl staining. Sections were first dehydrated in increasing concentration of ethanol (70\%, 95\%, 100\%) and immersed in xylene (VWR). Slices were rehydrated with decreasing concentration of ethanol $(100 \%, 95 \%$, $70 \%, 50 \%)$ and stained with Cresyl echt violet solution (incubation at $60^{\circ} \mathrm{C}$ for $13 \mathrm{~min}$, Abcam). Sections were then rinsed in $\mathrm{H}_{2} \mathrm{O}_{\text {dd }}$, dehydrated in ethanol and then mounted and secured with coverslip on microscope slides. Brain sections were scanned at $5 \times$ with an AxioScanZ1 slide scanning microscope (Carl Zeiss) and estimation of electrode positions was conducted using ZEN imaging light software (Carl Zeiss).

\section{Statistics}

All results are expressed as mean \pm SEM, error bars represent SEM and nonparametric statistics were adopted. In a repeated measure design with dependent variables having more than two levels, principal effect of the variable was tested with Friedmans test. 
Group comparisons in repeated measures design were tested with Wilcoxon signed-rank (S-R) test, otherwise Mann-Whitney test was adopted. Estimation statistics were also performed with the web application https:// www.estimationstats.com for group comparisons, and the effect size is reported in the figures as bootstrap resampling distribution of mean difference (bias-corrected and accelerated, 5000 resamples), with 95\% confidence interval $(\mathrm{Cl})$ represented by a bold black line below the distribution. In the text, the effect size is reported as mean difference [95\% Cl: lower bound, upper bound of the interval] (Ho et al., 2019). Linear fitting was performed with the least-square method and error bars were used as weights when averages across rats were fitted. To evaluate correlations and goodness of fit, the coefficient of determination $R^{2}$ was computed and $t$ test was performed to test the null hypothesis of slope $=0$, establishing a $p$ value. Gaussian $v$ test was used to test volume conduction in connectivity analysis (Cohen, 2014). All statistics are twotailed. The statistical significance in figures is represented as follows: ${ }^{*} p<0.05,{ }^{* *} p<0.01,{ }^{* * *} p<0.001$, $p \geq 0.05$ ns (not significant).

\section{Data and materials availability}

The code for computation of $\mathrm{PCl}^{\mathrm{ST}}$ is available in GitHub at the following link: github.com/renzocom/ PClst. Electrophysiological data used for analysis are available in EBRAINS at the following DOls: https://doi. org/10.25493/SODM-BK5 and https://doi.org/10.25493/ 5ZJY-PHB.

\section{Results}

\section{Single pulse electrical stimulation triggered complex ERPs during wakefulness but not during propofol anesthesia}

We recorded epidural EEG activity from 16 screw electrodes chronically implanted through the skull in head-restrained and body-restrained male, adult rats. Recording electrodes were in contact with the dura and organized in a symmetric grid, covering most of the cortex in both hemispheres (M2; M1; S1; RS; PA; V1; V2; GND). We stimulated right $\mathrm{M} 2$ by single monophasic, electrical current pulses (typically: 1-ms duration, 50- $\mu \mathrm{A}$ amplitude, at $0.1 \mathrm{~Hz}$ ) via a chronically implanted bipolar electrode, located $4.38 \pm 0.26 \mathrm{~mm}$ rostral from bregma, $0.47 \pm 0.09$ $\mathrm{mm}$ below the cortical surface (based on histology after recording, eight rats; Fig. $1 A$ ). The tips of the bipolar electrode were mainly located within Layer II/III across rats. However, one rat had one of the tips of the bipolar electrode placed in Layer I ( $0.05 \mathrm{~mm}$ from cortical surface), while another rat had one tip of the bipolar electrode placed in the upper part of Layer V $(0.92 \mathrm{~mm}$ from cortical surface). Pulse trains delivered at similar rostral-caudal coordinates triggered coordinated whisker deflections (Brecht et al., 2004), whereas EEG responses following single stimuli were not measurably contaminated by movements (Extended Data Fig. 1-1) and were reproducible throughout recording sessions and across days
(Extended Data Fig. 1-2). No correlation between the stimulating electrode locations and ERP amplitude or duration was found (Extended Data Fig. 1-3).

We performed electrophysiological recordings in nine rats during wakefulness and propofol anesthesia $(\sim 1.1 \mathrm{mg} / \mathrm{kg} / \mathrm{min}$, i.v. $)$ at a depth that produced spontaneous, slow, high-amplitude EEG oscillations and was sufficient to abolish any detectable motor response to pain stimuli. The redistribution of EEG power from high to low frequencies was confirmed by a reduced spectral exponent of the periodrogram (range: $20-40 \mathrm{~Hz}$ ) from wakefulness to propofol anesthesia (wakefulness: $-1.44 \pm 0.12$, propofol: $-3.12 \pm 0.09$; Wilcoxon S-R test, $p=0.004$; Fig. $1 B$ ). During wakefulness, single pulse stimulation $(1 \mathrm{~ms}, 50 \mu \mathrm{A})$ triggered long-lasting ERPs, including an early, fast, high-voltage response followed by multiple changes in polarity over time and across cortical areas. During anesthesia, however, the same stimulation produced only a similar initial activation, followed by fewer polarity changes (Fig. 1C,D). The ERP complexity was quantified by $\mathrm{PCl}^{\mathrm{ST}}$, a version of $\mathrm{PCl}$ based on the state transitions of principal components of the EEG response (Comolatti et al., 2019; Fig. 1E; see Materials and Methods). We initially assessed the $\mathrm{PCl}^{S T}$ time course, using sliding windows of $0.1 \mathrm{~s}$. Immediately after stimulation, $\mathrm{PCl}^{\mathrm{ST}}$ was similar across conditions and quickly decayed. Soon afterward, however, complexity $\left(\mathrm{PCl}^{S T}\right)$ built up reaching a maximum at $0.21 \pm 0.04$ s during wakefulness, and differed significantly from propofol anesthesia starting from $0.08 \mathrm{~s}$ until the ERP ended (Wilcoxon S-R test; from $0.62 \mathrm{~s}$, the $\mathrm{PCl}^{S T}$ values alternated between being significantly different and not significantly different, while from $0.84 \mathrm{~s}$, we found no further period with statistically significant differences until $1.1 \mathrm{~s}$, with the exception of a transient difference at $0.94 \mathrm{~s}$; Fig. $1 F$ ). Thus, in the time window $0.08-0.6 \mathrm{~s}, \mathrm{PCl}^{S T}$ showed a clear reduction from wakefulness to propofol anesthesia, for both single rats and the population (wakefulness: $42.35 \pm 3.47$, propofol: $6.63 \pm 1.38$; mean difference $=$ -35.7 [95\% Cl: $-41.16,-29.93]$; Wilcoxon S-R test, $p=0.004$; Fig. $1 F)$. In the same time range, the reduced $\mathrm{PCl}^{S T}$ was determined both by a reduced number of principal components (wakefulness: $4.44 \pm 0.18$, propofol: $1.55 \pm 0.24$; mean difference $=-2.89[95 \% \mathrm{Cl}$ : -3.44, -2.33]; Wilcoxon S-R test, $p=0.004$; Fig. 1G) and a reduced NST over time (wakefulness: $9.42 \pm 0.48$, propofol: $4.14 \pm 0.57$; mean difference $=-5.28[95 \% \mathrm{Cl}$ : -6.25, -4.46]; Wilcoxon S-R test, $p=0.004$; Fig. $1 H$; Extended Data Fig. 1-4).

\section{A period of HF suppression preceded the early interruption of deterministic response during propofol anesthesia}

Next, we quantified the changes in spectral power caused by the stimulation, within a HF range $(20-40 \mathrm{~Hz})$ that has been shown to maximize the difference between active and silent periods of a neuronal network that oscillates between depolarized and hyperpolarized states (Steriade et al., 1993, 1996, 2001; Mukovski et al., 2007), 
A

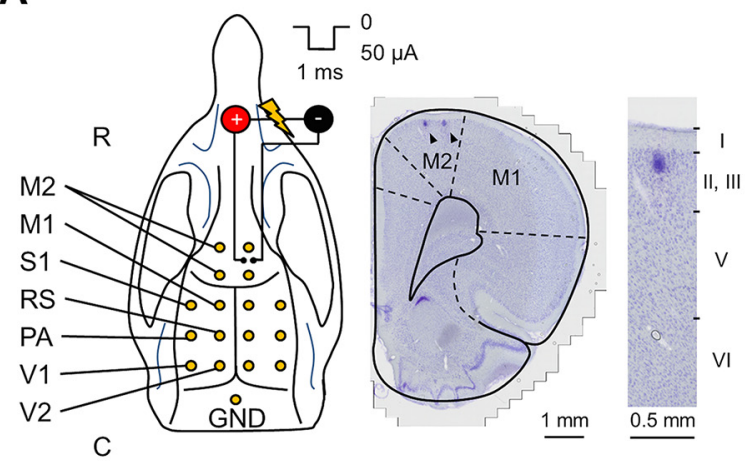

B

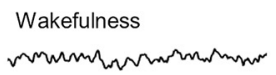

Propofol
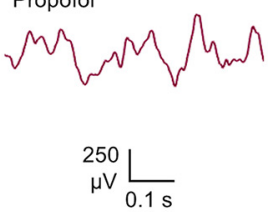

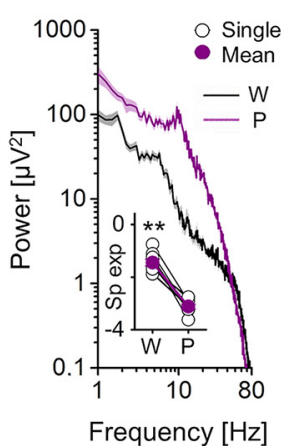

C Wakefulness Propofol
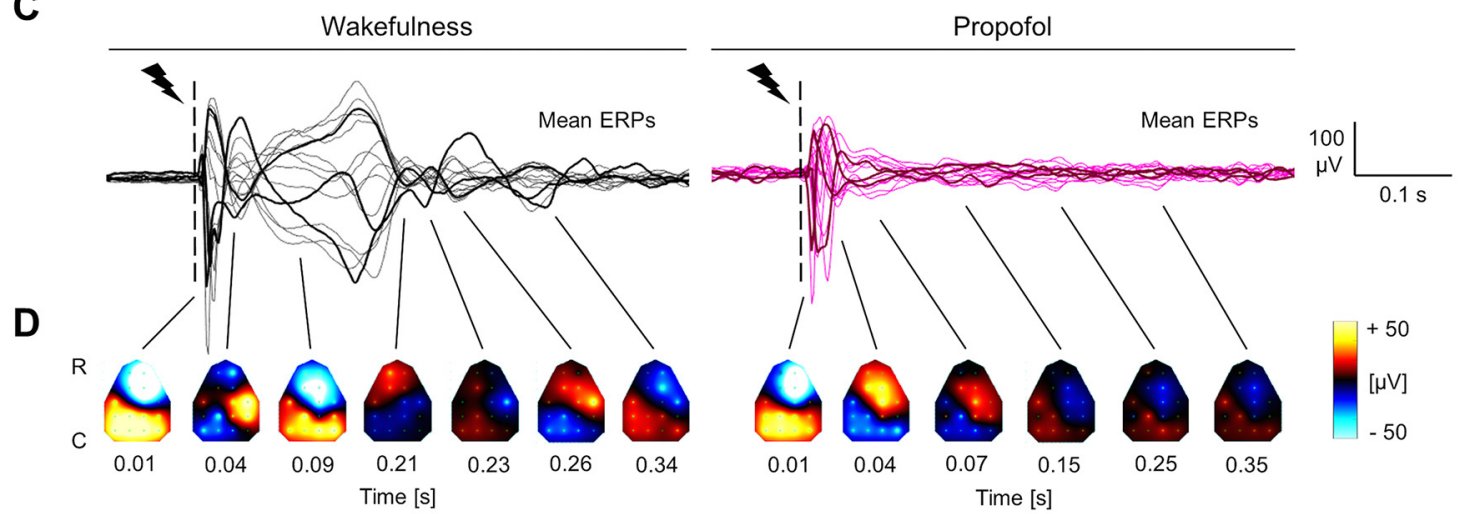

E
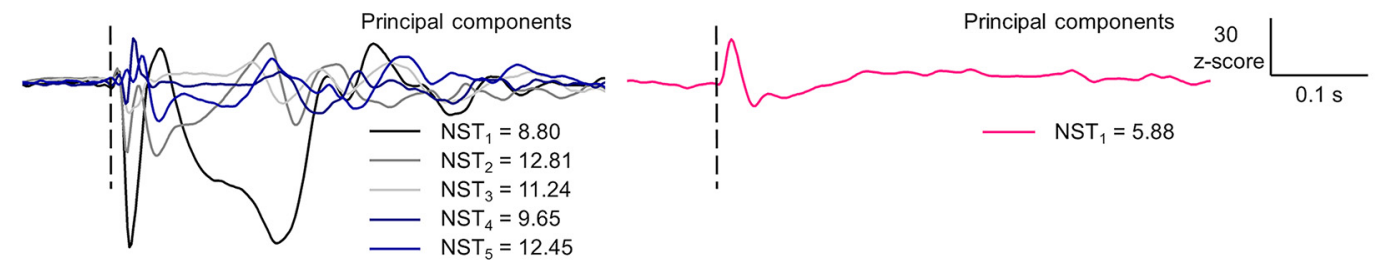

$\mathbf{F}$

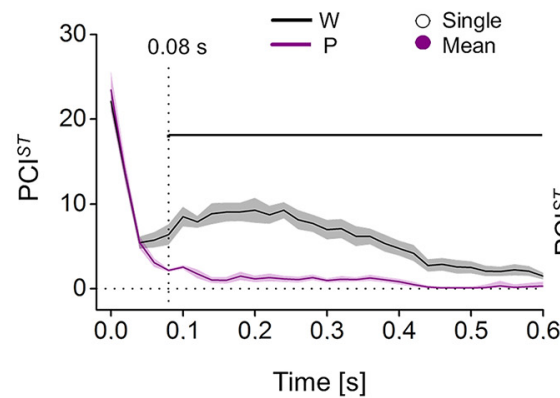

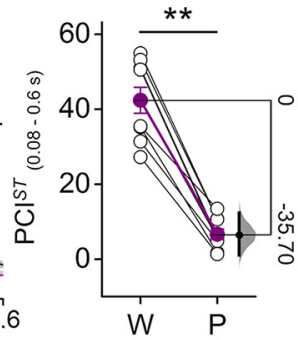

Condition
G

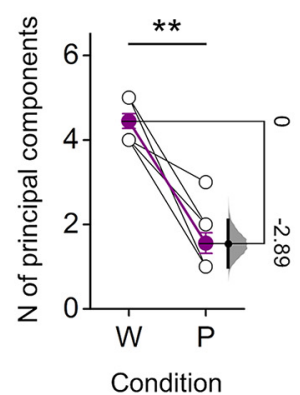

H

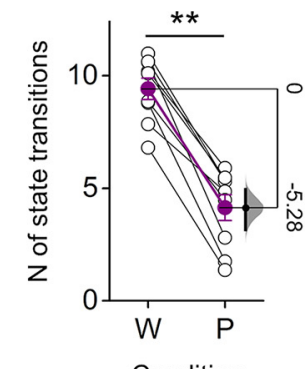

Figure 1. Spatiotemporal dynamics of evoked responses to electrical stimulation of $M 2$ during wakefulness and propofol anesthesia. $\boldsymbol{A}$, left, Positions in the rat skull of the 16 screw electrodes (yellow dots) and bipolar stimulating electrode used for recording EEG and triggering ERPs (R-C: rostral-caudal). Right, Coronal brain section (Nissl staining) showing the location of stimulating electrode in right M2. Black arrowheads indicate marks from the two poles of the bipolar electrode. Far right, Magnified view showing the site of one pole relative to cortical layers. B, left, Spontaneous EEG from one rat during wakefulness (W) and propofol (P) anesthesia. Right, Mean periodograms of spontaneous activity from one animal in the same conditions (shades represent SEM), and spectral exponents from all rats (inset). $\boldsymbol{C}-\boldsymbol{E}$, EEG responses to single pulse electrical stimulation ( $1 \mathrm{~ms}$, $50 \mu \mathrm{A}$; dashed line) from one rat during wakefulness and propofol anesthesia. Butterfly plots show superimposed mean ERPs from all recording electrodes (ERPs from three channels are in bold for clarity; $\boldsymbol{C}$ ) and their spatial distributions at different time points (interpolated ERPs, colorcoded; $\boldsymbol{D}$ ). $\boldsymbol{E}$, Derived principal components (from the same data as in $\boldsymbol{C}, \boldsymbol{D}$ ) with corresponding NST. $\boldsymbol{F}$, left, Time course of PCI ${ }^{S T}$ averaged from nine rats in wakefulness and propofol anesthesia (0 s: stimulus onset; shades represent SEM; horizontal line indicates statistical difference, $p<0.05)$. Right, $\mathrm{PCl}^{S T}$ quantified within the time window $0.08-0.60 \mathrm{~s}$. Number of principal components $(\boldsymbol{G})$ and average NST $(\boldsymbol{H})$ across conditions for all rats. The floating axis on the right of each simple group comparison (panels $\boldsymbol{F}$, right, $\mathbf{G}, \boldsymbol{H}$ ) shows the mean difference between conditions. The effect size is reported as bootstrap resampling distribution of mean 
continued

difference, with 95\% Cl represented by the bold black vertical line. See Extended Data Figure 1-1 for examination of possible whisker deflections induced by electrical stimulation. See Extended Data Figure 1-2 for assessment of the reproducibility of ERPs in time. See Extended Data Figure 1-3 for examination of possible correlation of ERP amplitude and length with stimulus location. See Extended Data Figure 1-4 for explanation of $\mathrm{PCl}^{S T}$ decomposition in number of principal components and state transitions.

as typically occurs during NREM sleep and general anesthesia (Steriade et al., 1993, 2001; Volgushev et al., 2006). We also considered the responses as "deterministic," i.e., reliably driven by the stimulation, if the evoked potentials were largely reproducible in phase when the same stimulation was repeated (David et al., 2006). Consequently, the duration of the deterministic neuronal response was defined as the last time point of the phase-locked component of the ERP measured as ITPC (Cohen, 2014), in 8- to 40-Hz frequency range (Pigorini et al., 2015; D'Andola et al., 2018; Rosanova et al., 2018).

Coherently with the $\mathrm{PCI}^{S T}$ time course, the ERP showed an early, transient increase in HF power during both wakefulness and propofol anesthesia, which was followed by a SC that corresponded to a transient increase in LF power (1-4 Hz; Fig. 2A; Extended Data Figs. 2-1, 2-2). A later $\mathrm{HF}$ activation, sustained until the end of the response in all channels, was detected only during wakefulness. By contrast, propofol anesthesia induced a deep HF suppression in most of the channels at $0.08 \pm 0.01 \mathrm{~s}$ (Fig. $2 A$, $B)$. When averaging the relative HF power during the suppression (0.08-0.18 s), across channels and rats, we found that it decreased from wakefulness to propofol anesthesia, to values below the baseline in all rats (wakefulness: $4.13 \pm 0.59 \mathrm{~dB}$, propofol: $-0.79 \pm 0.25 \mathrm{~dB}$; mean difference $=-4.92$ [95\% Cl: $-6.68,-3.77]$; Wilcoxon S-R test, $p=0.004$; Fig. 2C). Traces of HF suppression after stimulation were observed also during wakefulness, but these were briefer and shallower than with propofol (Extended Data Fig. 2-3), and were not seen after averaging (Fig. 2B,C). Moreover, during wakefulness the stimulation evoked durable, phase-locked responses in all electrodes. In contrast, during propofol anesthesia the period with HF suppression preceded an earlier drop of ITPC in all channels, followed by transient and not phaselocked HF activations in few cortical areas (Fig. 2A,B). Consequently, the phase-locked response, measured by averaging the ITPC drop time across channels and rats, was significantly briefer during propofol $(0.13 \pm 0.01 \mathrm{~s})$ than during wakefulness $(0.32 \pm 0.04 \mathrm{~s}$; mean difference $=-0.19[95 \% \mathrm{Cl}:-0.25,-0.14]$; Wilcoxon S-R test, $p=0.004$; Fig. 2D).

Next, we increased the stimulation intensity from 40 to $100 \mu \mathrm{A}$ (1 ms; five rats), attempting to compensate for the inhibiting effect of propofol (Ouyang et al., 2003; Bieda and Maclver, 2004; Fig. 3A). The resulting neuronal excitation, quantified by the rms amplitude of the first deflections of mean ERPs (early ERP rms, up to $0.05 \mathrm{~s}$ ), increased linearly with stimulus intensity, during both wakefulness (Friedman test, $p=0.002$; linear fit, $p=0.006$, $R^{2}=0.988$; Fig. $3 B$ ) and propofol anesthesia (Friedman test, $p=0.002$; linear fit, $p=0.016, R^{2}=0.968$ ). Overall, no significant differences in Early ERP rms were detected between wakefulness and anesthesia (Friedman test, $p=0.917)$. With propofol, the increased excitation was accompanied by a deeper HF suppression as indicated by the linear decrease of HF power as function of stimulus intensity (Friedman test, $p=0.005$; linear fit, $p=0.015, R^{2}=$ 0.969; Fig. $3 A, C)$ and the most negative peak within the period of HF suppression (HF suppression max) highly correlated in magnitude with the maximal amplitude of the SC of the ERP (SC max; Fig. 3D; linear fit, $p=2.809 \times$ $10^{-4}, R^{2}=0.529$; average values across channels for each rat and stimulus intensity). In contrast, during wakefulness, the mean HF power was always above baseline, thus higher than during propofol (Friedman test, $p=1.767 \times 10^{-7}$; Fig. $3 C$ ), and no change with stimulus intensity was detected (Friedman test, $p=0.178$; Fig. $3 C$ ). During propofol anesthesia, higher stimulus intensities were also linearly related to prolonged periods of HF suppression (Fig. $3 A$; Extended Data Fig. 2-3; Friedman test, $p=0.005$; linear fit, $p=0.024, R^{2}=0.953$ ). In contrast, we did not find intensity dependent change in the brief HF suppressions during wakefulness (Extended Data Fig. 2-3). We then used the changes in duration seen with propofol to assess a temporal relation between the interruption of ITPC and HF suppression. We found a first significant correlation with the latency of HF suppression max (linear fit, $p=0.043, R^{2}=$ 0.209 ; average values across channels for each rat and stimulus intensity; Fig. $3 E$ ) and a stronger temporal correlation with the end of HF suppression (linear fit, $p=0.008, R^{2}$ $=0.333$; average values across channels for each rat and stimulus intensity; Extended Data Fig. 3-1). Interestingly, on average across channels and rats, the end time point of HF suppression was coincident with the latency of SC max (Extended Data Fig. 2-1). Finally, $\mathrm{PCl}^{S T}$ was always higher and ITPC more long-lasting during wakefulness than with propofol, regardless of the stimulus intensity, in all tested animals (Extended Data Fig. 3-2).

\section{ERPs during ketamine anesthesia showed intermediate complexity}

In order to test whether the $\mathrm{PCl}^{S T}$ reduction with propofol might be related to behavioral unresponsiveness per se, we repeated single pulse stimulations ( $1 \mathrm{~ms}, 50 \mu \mathrm{A}$; eight rats) during ketamine anesthesia, which was found to maintain high brain complexity in humans (Sarasso et al., 2015), at a dose that abolished all motor responses to painful stimuli $(\sim 1.8 \mathrm{mg} / \mathrm{kg} / \mathrm{min}$ i.v.; Fig. $4 A)$. Similarly to wakefulness, the spontaneous EEG activity with ketamine showed fast, shallow oscillations, with similar spectral exponent (seven rats; wakefulness: $-1.21 \pm 0.24$, ketamine: $-0.97 \pm 0.09$; Wilcoxon S-R test, $p=0.156$; Fig. $4 B$ ). The $\mathrm{PCl}^{S T}$ time course revealed a similar initial complexity of the ERP across conditions that quickly decayed. Like wakefulness, but unlike propofol, during ketamine anesthesia $\mathrm{PCl}^{S T}$ increased soon after the initial decay, 
A

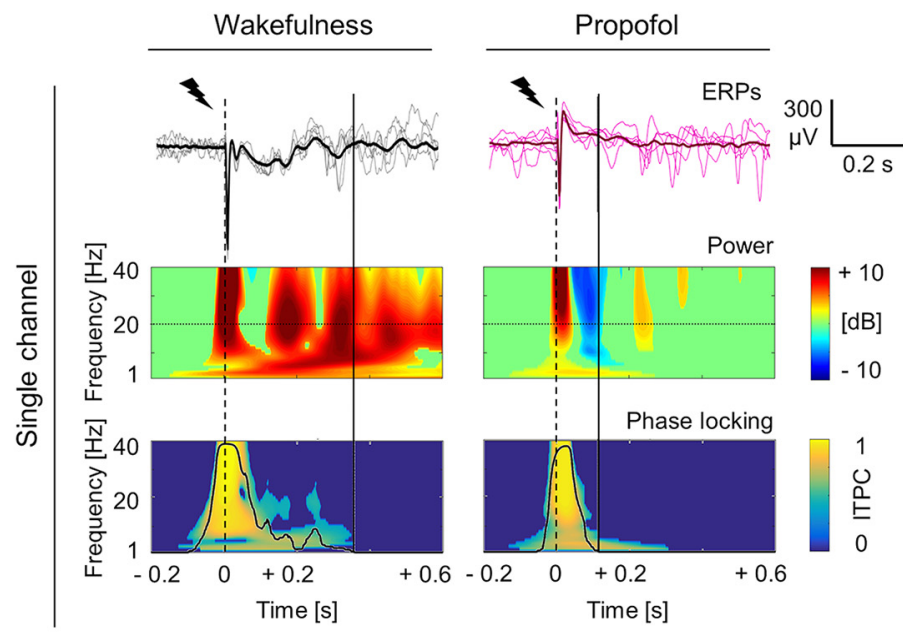

B

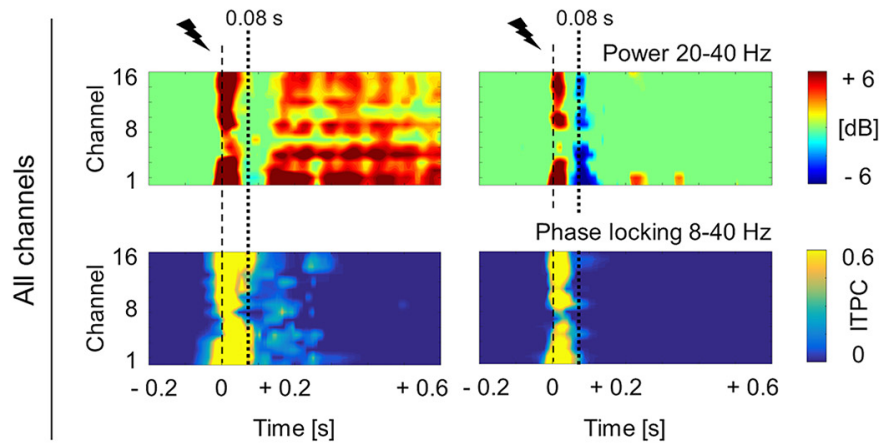

C

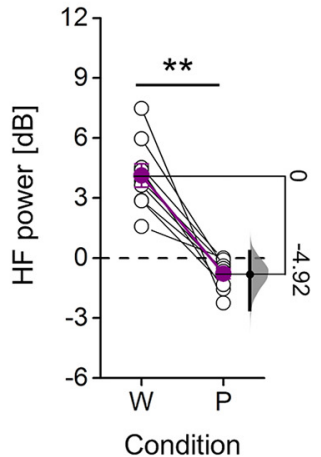

D

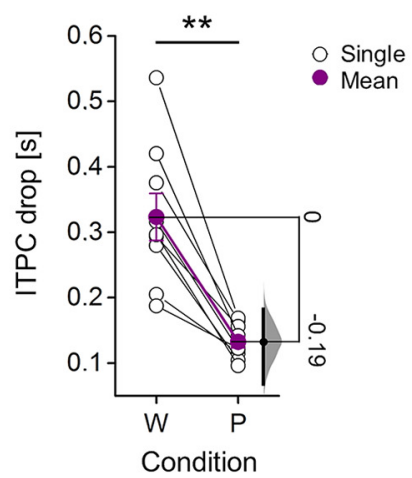

Figure 2. Propofol anesthesia induced suppression of high frequencies and reduced phase-locking in response to electrical stimulation, compared with wakefulness. $\boldsymbol{A}$, Example of epidural EEG response to single pulse electrical stimulation $(1 \mathrm{~ms}$, $50 \mu \mathrm{A}$; dashed line) from the same rat during wakefulness (left) and propofol anesthesia (right). The mean ERPs (bold) and five consecutive single trials from the same frontal channel (M2) are shown in both conditions (top) with relative spectrogram (middle) and ITPC (below) for all frequencies in range $1-40 \mathrm{~Hz}$. The temporal dynamic of averaged ITPC in range $8-40 \mathrm{~Hz}$ is represented by a black superimposed curve that goes from 0 to 1 (ordinate axis on the right, same values of color map). The continuous vertical lines indicate the time point of the drop of averaged ITPC in range $8-40 \mathrm{~Hz}$. B. Time course of the average HF power in the $20-$ to $40-\mathrm{Hz}$ range (top), and the averaged ITPC in the $8-$ to $40-\mathrm{Hz}$ range (below) plotted for all channels from the same rat and conditions of $\boldsymbol{A}$. The dotted vertical line at $0.08 \mathrm{~s}$ indicates the mean onset of HF suppression across rats during propofol anesthesia. The mean HF power (in time range: $0.08-0.18 \mathrm{~s} ; \boldsymbol{C})$ and the duration $(\boldsymbol{D})$ of phase-locking across trials (time of ITPC drop) for all animals $(n=9)$ during wakefulness $(\mathrm{W})$ and propofol anesthesia $(P)$. The floating axis on the right of each simple group comparison (panels $\boldsymbol{C}, \boldsymbol{D})$ shows the mean difference between conditions. The effect size is reported as bootstrap resampling distribution of mean difference, with $95 \% \mathrm{Cl}$ represented by the bold black vertical line. See Extended Data Figures 2-1, 2-2 for examination of single trial responses and SC of ERPs in propofol and wakefulness conditions, respectively. See Extended Data Figure 2-3 for in depth examination of HF suppression, comparing propofol anesthesia to wakefulness conditions. 
A
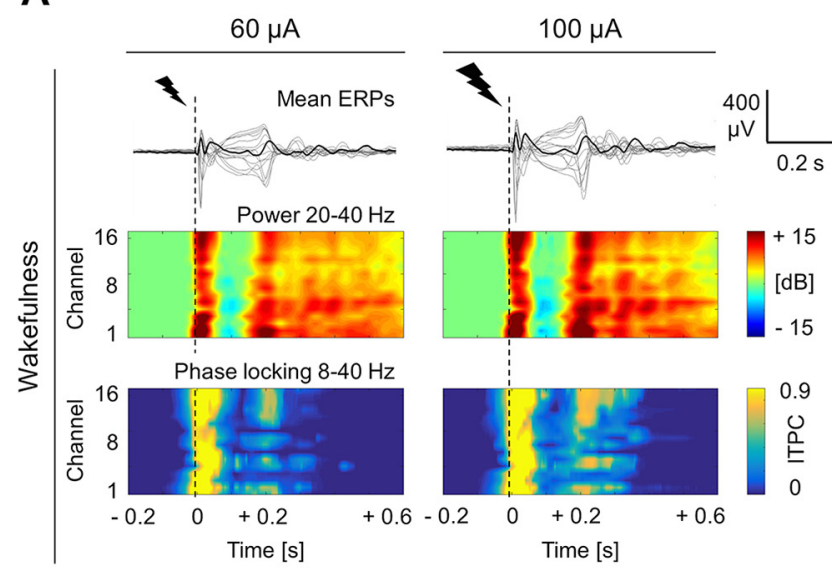

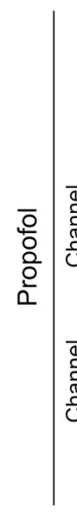
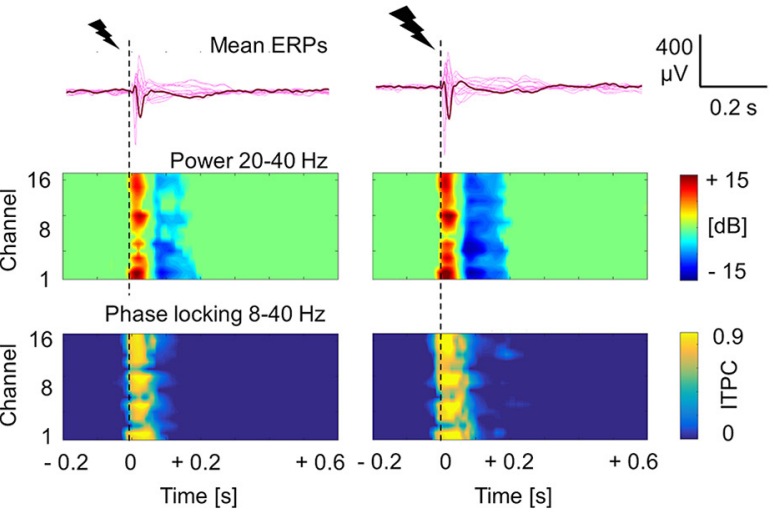

B

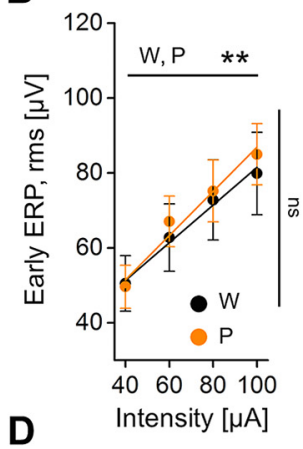

C

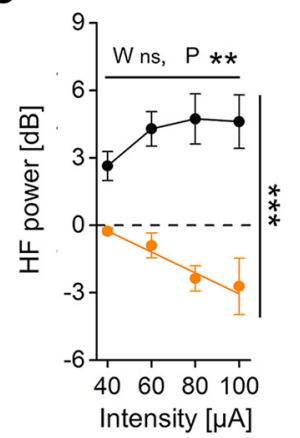

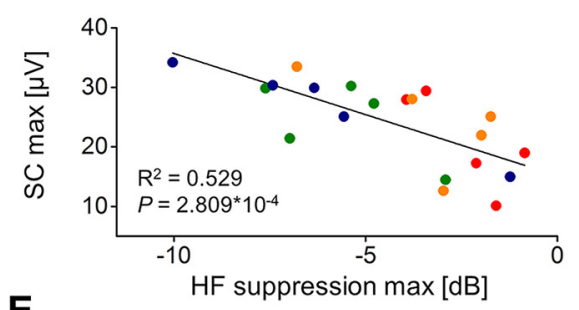

E

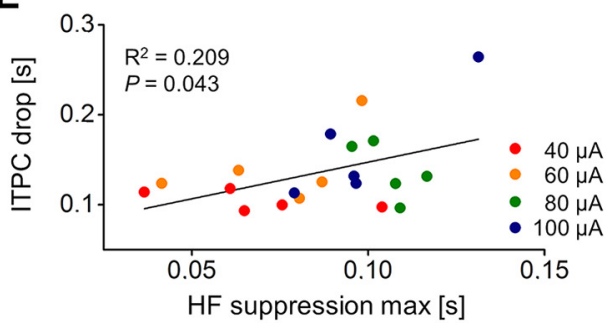

Figure 3. During propofol anesthesia, the HF suppression was deeper after stronger stimulation, and correlated with the SC of the ERP and with the drop in phase-locking. $\boldsymbol{A}$, Mean ERPs from the same rat in response to single pulse electrical stimulations (dashed lines) at two different intensities [60 $\mu \mathrm{A}$ (left); $100 \mu \mathrm{A}$ (right)], during wakefulness (top) and during propofol anesthesia (bottom). The butterfly plots show averaged ERPs from all recording electrodes superimposed, with one mean ERP from the same parietal channel (PA) shown in bold for clarity. Below, The HF power $(20-40 \mathrm{~Hz})$ and the ITPC $(8-40 \mathrm{~Hz})$ for all channels are shown. $\boldsymbol{B}$, Quantification of early ERP rms amplitude (up to $0.05 \mathrm{~s} ; \boldsymbol{B}$ ) and HF power (in range 0.08-0.18 s; $\boldsymbol{C}$ ) as a function of increasing stimulation intensity during both wakefulness $(\mathrm{W})$ and propofol anesthesia $(\mathrm{P})$. Values are averaged across channels and animals $(n=5)$. $\boldsymbol{D}$, During propofol anesthesia, the maximal value of HF suppression (negative peak of HF power in range 0-0.3 s) correlated in magnitude with the maximal absolute amplitude of the SC of the ERP (in range 0-0.6 s). $\boldsymbol{E}$, The maximal HF suppression also correlated in time with the drop of phase-locking across trials (ITPC drop). The averaged values across channels are plotted for each rat and stimulus intensity (color-coded). The coefficient of determination $\mathrm{R}^{2}$ and the $p$ value are reported. See Extended Data Figure 3-1 for examination of correlations between ITPC drop time and different time points of HF suppression. See Extended Data Figure 3-2 for analysis of $\mathrm{PCl}^{\mathrm{ST}}$ and ITPC drop time in relation to increasing stimulus intensity.

reaching a peak at $\sim 0.2 \mathrm{~s}$ and gradually decreased until the ERP end. From $0.08 \mathrm{~s}$ the complexity level with ketamine was lower than during wakefulness, but transient periods of similar $\mathrm{PCl}^{\mathrm{ST}}$ were detected (Wilcoxon S-R test; from $0.44 \mathrm{~s}$ we found no significant differences between wakefulness and ketamine conditions until $1.1 \mathrm{~s}$, except an isolated moment of difference at $0.62 \mathrm{~s}$; Fig. $4 C)$. Similarly, from $0.16 \mathrm{~s}, \mathrm{PCl}^{S T}$ with ketamine was significantly higher than during propofol anesthesia and only brief periods of similar complexity were identified until the end of ERP (Mann-Whitney test; Fig. 4C). Coherently, $\mathrm{PCl}^{S T}$ differed between wakefulness and ketamine anesthesia for the 0.08-0.6 s period (wakefulness: $41.80 \pm 5.17$, ketamine: $21.14 \pm 4.48$; mean difference $=$ -20.66 [95\% Cl: -26.60, -14.80]; Wilcoxon S-R test, $p=0.008$; Fig. 4C), but also between ketamine and propofol anesthesia (mean difference $=-14.51[95 \% \mathrm{Cl}$ :
-23.02, -6.13]; Mann-Whitney test, $p=0.024$; Fig. 4C). The intermediate $\mathrm{PCl}^{S T}$ with ketamine was explained by a similar number of ERP components compared with wakefulness (wakefulness: $4.37 \pm 0.18$, ketamine: $3.62 \pm 0.62$; mean difference $=-0.75[95 \% \mathrm{Cl}:-1.87$, 0]; Wilcoxon S-R test, $p=0.25$; Fig. 4D), with a reduced NST (wakefulness: $9.36 \pm 0.81$, ketamine: $5.50 \pm 0.31$; mean difference $=-3.86[95 \% \mathrm{Cl}$ : $-5.00,-2.63]$; Wilcoxon S-R test, $p=0.008$; Fig. $4 E$; see also Extended Data Fig. 1-4). After the similarly complex initial response, also with ketamine a period of HF suppression occurred at $0.08 \pm 0.01 \mathrm{~s}$ (wakefulness: $4.25 \pm 0.78 \mathrm{~dB}$, ketamine: $-1.47 \pm 0.44 \mathrm{~dB}$, mean difference $=-5.72[95 \% \mathrm{Cl}:-7.71,-4.25]$; Wilcoxon S-R test, $p=0.008$; Fig. $4 F$ ), but no consistent changes in ITPC drop time were found (wakefulness: $0.32 \pm 0.04 \mathrm{~s}$, ketamine: $0.23 \pm 0.04 \mathrm{~s}$; mean difference $=-0.09[95 \%$ 
A

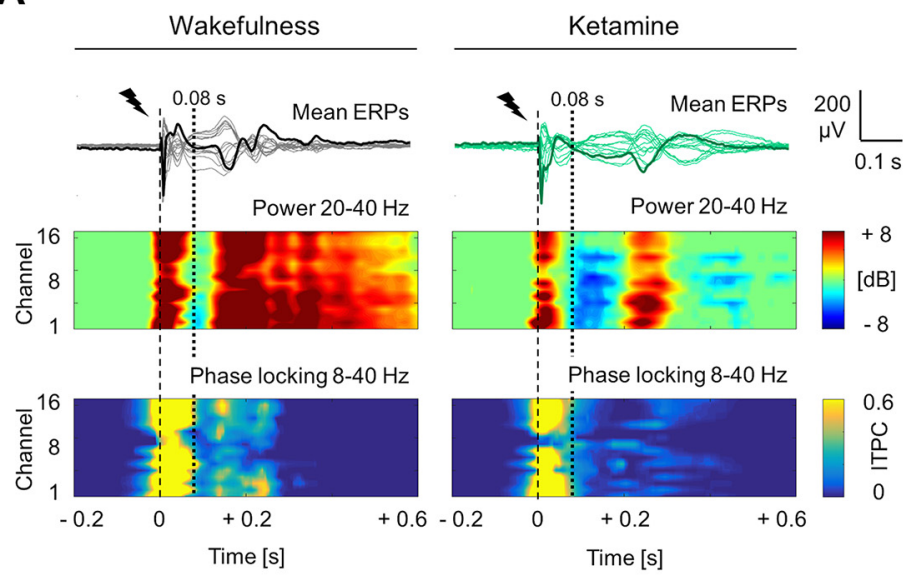

C

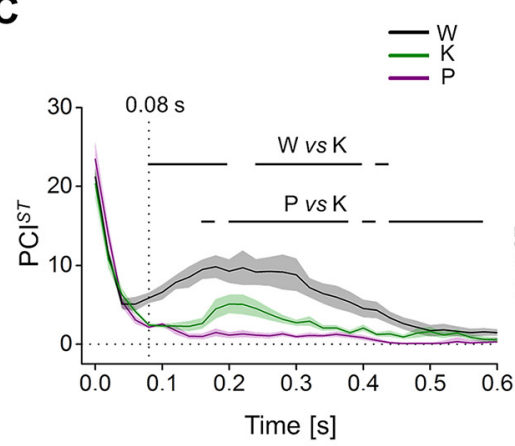

B
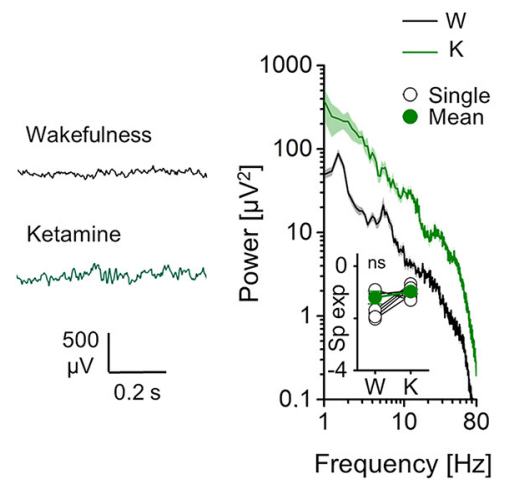

E
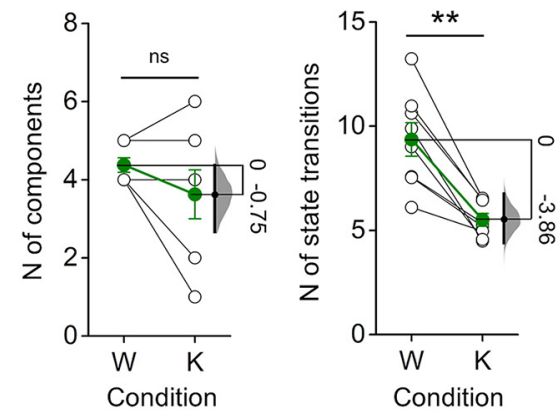

F

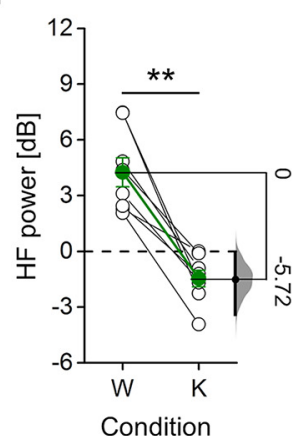

G

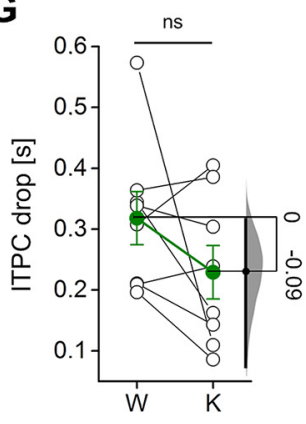

Condition

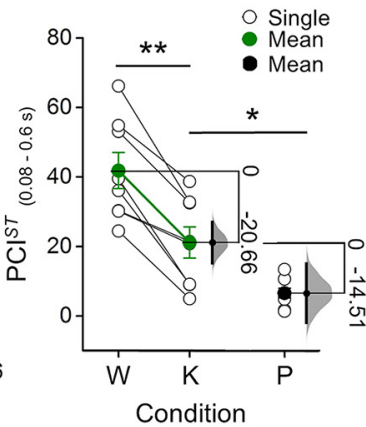

H

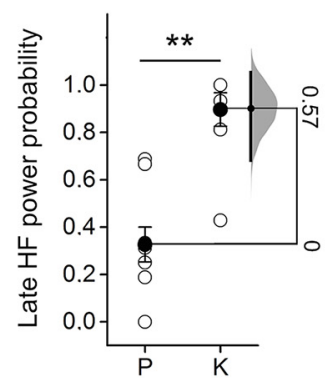

Condition

I

$\rightarrow$ Late HF power onset

$\bullet$ ITPC drop 8-40 Hz

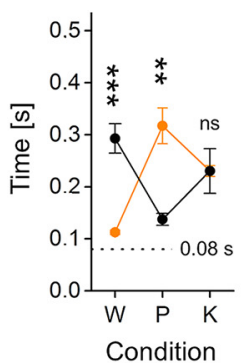

Figure 4. ERPs with ketamine showed intermediate $\mathrm{PCI}^{S T}$, with HF suppression, but sustained ITPC. $\boldsymbol{A}$, Mean ERPs from all electrodes in response to single pulse stimulation ( $1 \mathrm{~ms}, 50 \mu \mathrm{A}$; dashed line) shown superimposed, from the same rat during wakefulness and ketamine anesthesia. One averaged ERP from the same channel (M2) is in bold for clarity. Spectrograms of HF power and ITPC for all channels are shown below. Vertical dotted line at $0.08 \mathrm{~s}$ indicates the average time of onset of HF suppression. B, Spontaneous EEG (left) and relative mean periodograms (shades represent SEM; right) are shown from one rat during wakefulness $(\mathrm{W})$ and ketamine anesthesia (K). Spectral exponents from all rats are also shown (inset). C, left, Time courses of mean $\mathrm{PCI}^{\mathrm{T}}$ in wakefulness, ketamine, and propofol (P) anesthesia (shades represent SEM; horizontal lines indicate periods of statistical difference, $p<0.05$ ). Right, $P C I^{S T}$ in range $0.08-0.6 \mathrm{~s}$ is shown for each rat. Propofol data are the same as in Figure 1. D, Number of principal components and $\boldsymbol{E}$, average state transitions of EEG response are shown for all rats. Mean HF power (in range 0.08-0.18 s; $\boldsymbol{F}$ ) and time of ITPC drop averaged $(\boldsymbol{G})$ across channels are shown for all animals during wakefulness and ketamine anesthesia. $\boldsymbol{H}$, Ratio between the number of electrodes (channels) with a late increase in HF power (after $0.08 \mathrm{~s}$ ) and the total number of channels. $\boldsymbol{I}$, Temporal differences between the onset of the late HF power and ITPC drop are shown. The floating axis on the right of each simple group comparison (panels $\boldsymbol{C}$, right, to $\boldsymbol{H}$ ) shows the mean difference between conditions. The effect size is reported as bootstrap resampling distribution of mean difference, with $95 \% \mathrm{Cl}$ represented by the bold black vertical line. See Extended Data Figure 4-1 for in depth comparison with sevoflurane anesthesia.

Cl: -0.25 , 0]; Wilcoxon S-R test, $p=0.195$; Fig. $4 G$ ). After the period of HF suppression, a late increase in $\mathrm{HF}$ power occurred in a higher proportion of channels during ketamine anesthesia than with propofol (propofol,
$0.33 \pm 0.07 ;$ ketamine, $0.90 \pm 0.07 ;$ mean difference $=$ 0.57 [95\% Cl: $0.35,0.72]$; Mann-Whitney test, $p=$ 0.001 ; Fig. $4 H$ ), while in wakefulness, it was seen in all channels in all 12 animals. We then assessed whether 
this resumption of HF activity was still associated with the deterministic neuronal activation by comparing the time of ITPC drop with the onset of late HF power increment. Only channels that presented a late increase in HF power were included for this comparison. During wakefulness, the resumption of HF activity was largely deterministic, occurring within a period of significant ITPC (Wilcoxon S-R test, $p=4.883 \times 10^{-4}$; Fig. 4/), whereas during propofol the late HF power was not phase-locked as it occurred after the fading of ITPC (Wilcoxon S-R test, propofol, $p=0.008$; Fig. 4/). Coherently with the intermediate complexity level during ketamine, the late HF activity observed in this condition occurred with a variable relationship with respect to the drop of ITPC (Wilcoxon S-R test, $p=1$; Fig. 4/).

We also used another general anesthetic, the volatile sevoflurane, to test whether periods of HF suppression combined with reduced ITPC and low $\mathrm{PCl}^{S T}$ were not specific to propofol. Sevoflurane anesthesia in 10 rats produced results that resembled what we observed with propofol. Thus, the $\mathrm{PCl}^{S T}$ value was lower than during wakefulness and ketamine anesthesia, but no statistical difference was found when compared with propofol, and this low $\mathrm{PCl}^{S T}$ was explained by a reduced number of both principal ERP components and state transitions compared with wakefulness. A period of deep HF suppression $(-1.04 \pm 0.40 \mathrm{~dB})$ occurred at $0.075 \pm 0.005 \mathrm{~s}$ from stimulation and the phase-locked response dropped soon afterward, at $0.12 \pm 0.01 \mathrm{~s}$. As for propofol and differently from wakefulness and ketamine conditions, only few channels showed later increments of HF power, which were consistently not phase-locked (Extended Data Figs. 1-4, 4-1).

\section{Functional connectivity and diversity of response were conserved during wakefulness and ketamine anesthesia but collapsed with propofol and sevoflurane}

In principle, $\mathrm{PCl}$ estimates integration and differentiation in a neuronal network (Casali et al., 2013; Comolatti et al., 2019). Thus, high PCl value should indicate a highly connected network with diversified connectivity patterns. In order to test this, we assessed the functional connectivity across cortical regions following electrical stimulation by computing the ISPC (Cohen, 2014) for each channel pair, averaged in the $\theta$ - $\alpha$ frequency range (5$14 \mathrm{~Hz}$ ), which includes the frequency bands that showed more long-lasting ITPC in wakefulness (mean ITPC drop time across channels and 12 rats, for each frequency band $>4 \mathrm{~Hz}$ : $\theta=0.34 \pm 0.02 \mathrm{~s} ; \alpha=0.29 \pm 0.03 \mathrm{~s} ; \beta=$ $0.19 \pm 0.02 \mathrm{~s} ; \gamma=0.13 \pm 0.02 \mathrm{~s})$. We then averaged the resulting ISPC in two time windows of interest: during the HF suppression (0.08-0.18 s) and afterward, until the mean ITPC drop time in wakefulness, post-HF suppression $(0.18-0.3 \mathrm{~s})$. We considered the increase in ISPC above the baseline and computed the CD for each electrode, as the ratio between the number of significantly synchronized channel pairs over the total number of channels (Fig. 5A). During wakefulness, the averaged CD was sustained up to $0.3 \mathrm{~s}$ and was higher than during propofol anesthesia in both time windows (nine rats; HF suppression, wakefulness: $0.73 \pm 0.03$, propofol: $0.39 \pm 0.06$, mean difference $=-0.34$ [95\% Cl: -0.42 , $-0.25]$; post-HF suppression, wakefulness: $0.62 \pm 0.04$, propofol: $0.16 \pm 0.02$, mean difference $=-0.46[95 \% \mathrm{Cl}$ : $-0.54,-0.41]$; Wilcoxon S-R test, HF suppression: $p=$ 0.002 ; post-HF suppression: $p=0.004$; Fig. $5 B$ ). Sevoflurane gave similar results (nine rats; Extended Data Fig. 5-1). In contrast, ketamine anesthesia induced a significant drop of CD compared with wakefulness only during the HF suppression (seven rats; wakefulness: $0.75 \pm 0.04$, ketamine: $0.55 \pm 0.04$; mean difference $=-0.20$ [95\% Cl: $-0.36,-0.10]$; Wilcoxon S-R test, $p=0.031$; Fig. $5 C$ ). After the period of HF suppression, no significant difference was identified (wakefulness: $0.69 \pm 0.03$, ketamine: $0.59 \pm 0.05$; mean difference $=-0.11$ [95\% Cl: $-0.22,-0.03]$; Wilcoxon S-R test, $p=0.094)$. The absence of statistical difference in the $\theta$ - $\alpha$ frequency range was mainly explained by the connectivity in the $\theta$ band (5-7 Hz; Extended Data Fig. 5-2). Besides, we did not find statistical difference between wakefulness and ketamine conditions also in the LF range (1-4 Hz; Extended Data Fig. 5-2). Coherently, CD after the period of HF suppression was higher with ketamine than with both propofol and sevoflurane, while no difference was detected between these latter conditions (Mann-Whitney test, ketamine vs propofol, $p=0.001$; ketamine vs sevoflurane, $p=0.004$; Wilcoxon $S-R$ test, propofol vs sevoflurane, $p=0.375)$. We then identified a highly significant positive correlation between mean $\mathrm{CD}_{(0.18-0.3 \mathrm{~s})}$ across channels and $\mathrm{PCl}^{\mathrm{ST}}{ }_{(0.08-0.6 \mathrm{~s})}$, thus revealing a connection between functional connectivity and perturbational complexity (linear fit, $p=5.197 \times 10^{-10}, R^{2}=0.683$; Fig. $5 D$ ).

Not only the overall amount of connectivity differed between conditions; averaging $\mathrm{CD}_{(0.18-0.3 \mathrm{~s})}$ across channels organized in cortical regions (frontal: M2, M1; parietal: S1, PA, RS; occipital: V2, V1), revealed an uneven spatial distribution of $C D$ during both wakefulness and ketamine anesthesia (wakefulness, 11 rats, Friedman test, $p=0.019$; ketamine, 7 rats, Friedman test, $p=0.018$; Fig. $5 E$ ), indicating a peak of connectivity in the occipital region. For each region, $C D$ was similar between wakefulness and ketamine anesthesia (Mann-Whitney test, frontal, $p=0.717$; parietal, $p=0.526$; occipital, $p=0.856$ ) and no relation with cortical areas was detected with both sevoflurane and propofol (nine rats, sevoflurane, Friedman test, $p=0.368$; propofol, Friedman test, $p=0.459$ ). This suggested a similar degree of diversity in the evoked response among cortical areas during both wakefulness and ketamine conditions, which collapsed with sevoflurane and propofol.

A complementary way to conceive the diversity in activity patterns induced by a stimulus is in relation to the specific site of stimulation. We took advantage of the variability, across rats, in the precise location of the stimulating electrode within $\mathrm{M} 2$, and tested for possible correlations with $\mathrm{PCl}^{S T}$. We did not find correlation with the position of the stimulating electrode along the rostro-caudal axis (in range: $3-5 \mathrm{~mm}$ from bregma; Extended Data Fig. 6-1), but we detected a strong and positive correlation of $\mathrm{PCl}^{S T}$ with the location along the dorso-ventral axis (range: $0.1-0.83 \mathrm{~mm}$ from cortical surface, from Layers I to upper Layer V, mainly within Layer II/III; Extended Data 
A
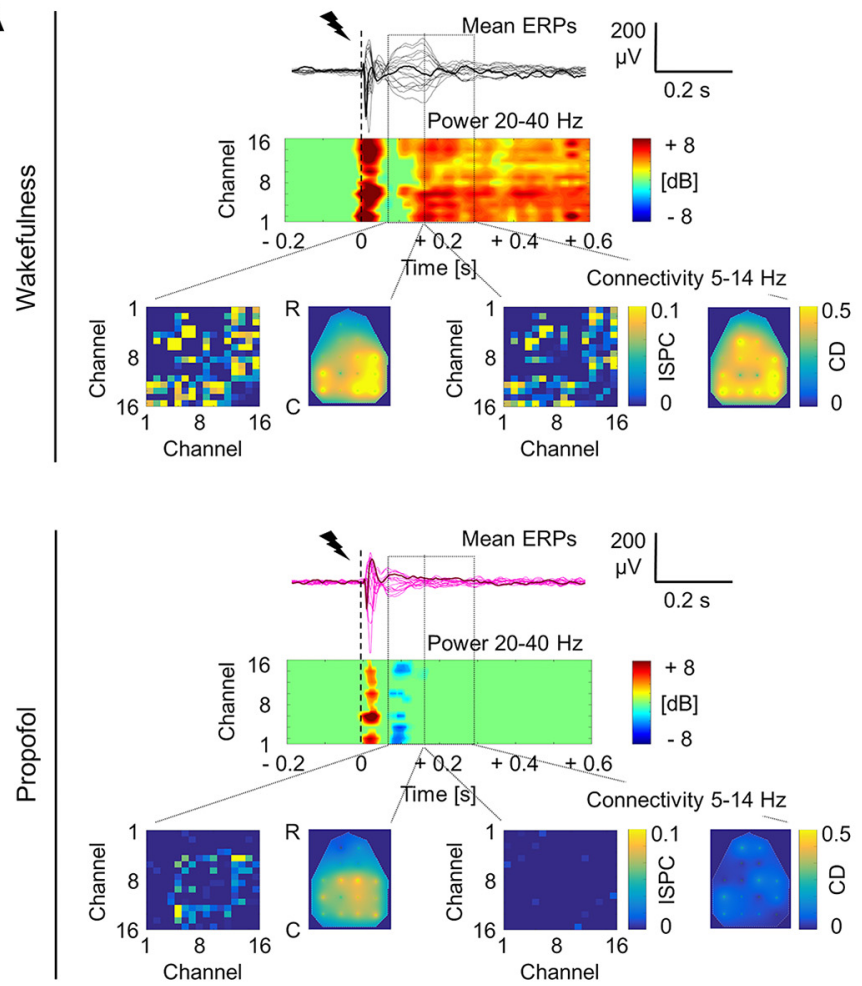



$\mid$

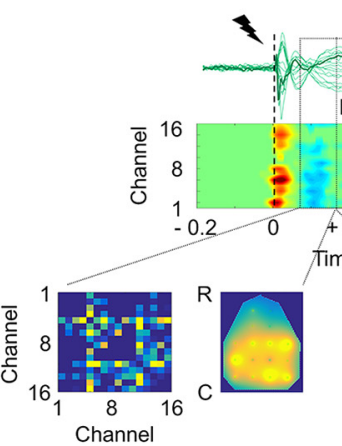

Mean ERPs

200

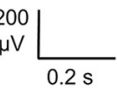

Power $20-40 \mathrm{~Hz}$

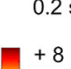

$5-14 \mathrm{~Hz}$

$+0.2+0.4+0.6$

Connectivity $5-14 \mathrm{~Hz}$

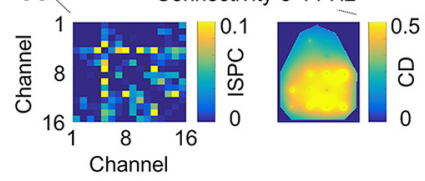

B

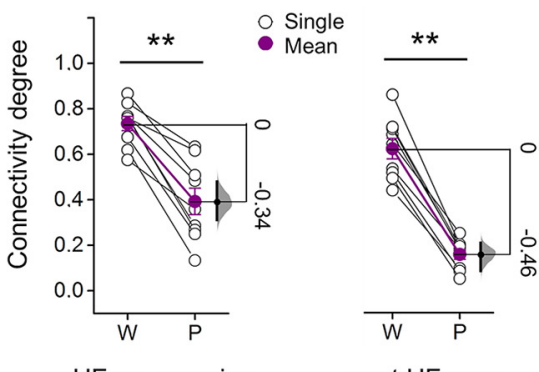

HF suppression

post HF supp.

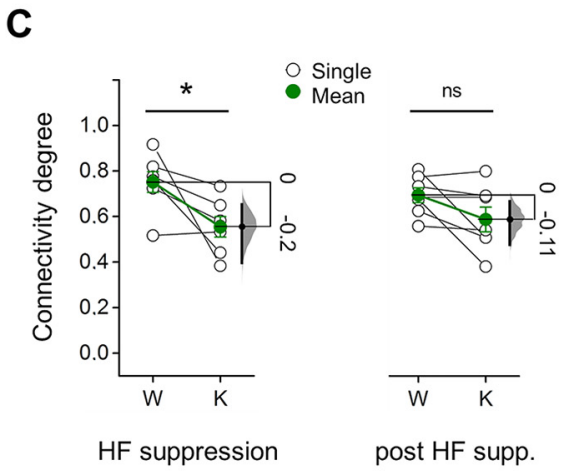

D

E
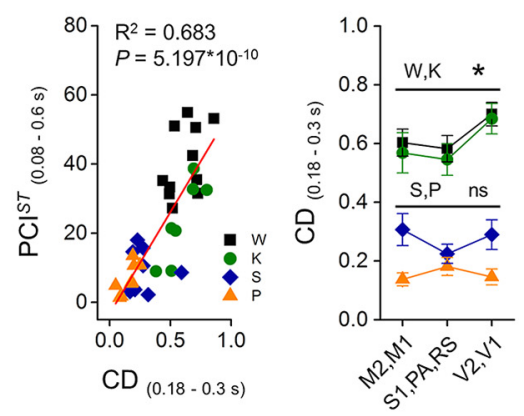

Figure 5. Functional cortical connectivity after perturbation was reduced during propofol or sevoflurane anesthesia compared with wakefulness, while was conserved with ketamine. $\boldsymbol{A}$, Superimposition of mean ERPs from all electrodes in response to single pulse stimulation ( $1 \mathrm{~ms}, 50 \mu \mathrm{A}$; dashed line) from the same rat during wakefulness $(\mathrm{W})$ and propofol $(\mathrm{P})$ and ketamine $(\mathrm{K})$ anesthesia (from up to bottom). One averaged ERP is shown in bold for clarity. Spectrograms of HF power are shown for each channel, below the butterfly plots. The bottom part of each inner panel reports increments in functional connectivity compared with baseline in two time windows: during HF suppression, 0.08-0.18 s (left) and after HF suppression, 0.18-0.3 s (right; rectangles indicate the time windows). For each window, the connectivity matrix based on mean ISPC $(5-14 \mathrm{~Hz})$ is reported on the left and the topographical distribution (R-C: rostral-caudal) of CD for each channel is interpolated and shown on the right. $\boldsymbol{B}, \boldsymbol{C}$, Mean CD across channels during HF suppression (left) and post-HF suppression (right) from rats during wakefulness and propofol $(\boldsymbol{B}, n=9)$, and ketamine $(\boldsymbol{C}, n=7)$ anesthesia. $\boldsymbol{D}$, Mean CD (range: $0.18-0.3 \mathrm{~s}$ ) across channels from all animals and conditions are plotted against PCI ${ }^{S T}$ (range: $0.08-$ $0.6 \mathrm{~s}$ ) and linearly fitted (coefficient of determination $R^{2}$ and $p$ value are reported). $\boldsymbol{E}$, Mean CD (range: $\left.0.18-0.3 \mathrm{~s}\right)$ across rats and across channels organized in three cortical regions are shown for each condition. In $\boldsymbol{D}$, $\boldsymbol{E}$, sevoflurane condition (S) is also reported $(n=9)$. The floating axis on the right of each simple group comparison (panels $\boldsymbol{B}, \boldsymbol{C})$ shows the mean difference between conditions. The effect size is reported as bootstrap resampling distribution of mean difference, with $95 \% \mathrm{Cl}$ represented by the bold black vertical line. See Extended Data Figure 5-1 for analysis of functional connectivity with sevoflurane anesthesia and Extended Data Figure 5-2 for analysis of functional connectivity across conditions for each frequency band of interest.

Fig. 6-1). Specifically, during wakefulness $\mathrm{PCl}^{\mathrm{ST}}{ }_{(0.08-0.6 \mathrm{~s})}$ positively correlated with the depth of the stimulation (linear fit, $p=0.003, R^{2}=0.908$; Fig. $6 A, B$ ) among six rats and a similar correlation was detected with ketamine (linear fit, $p=0.002, R^{2}=0.922$ ). This was explained by the increasing number of both principal components and state transitions with stimulation depth, in both wakefulness and ketamine conditions (Extended Data Fig. 6-2). 
A

Stim location
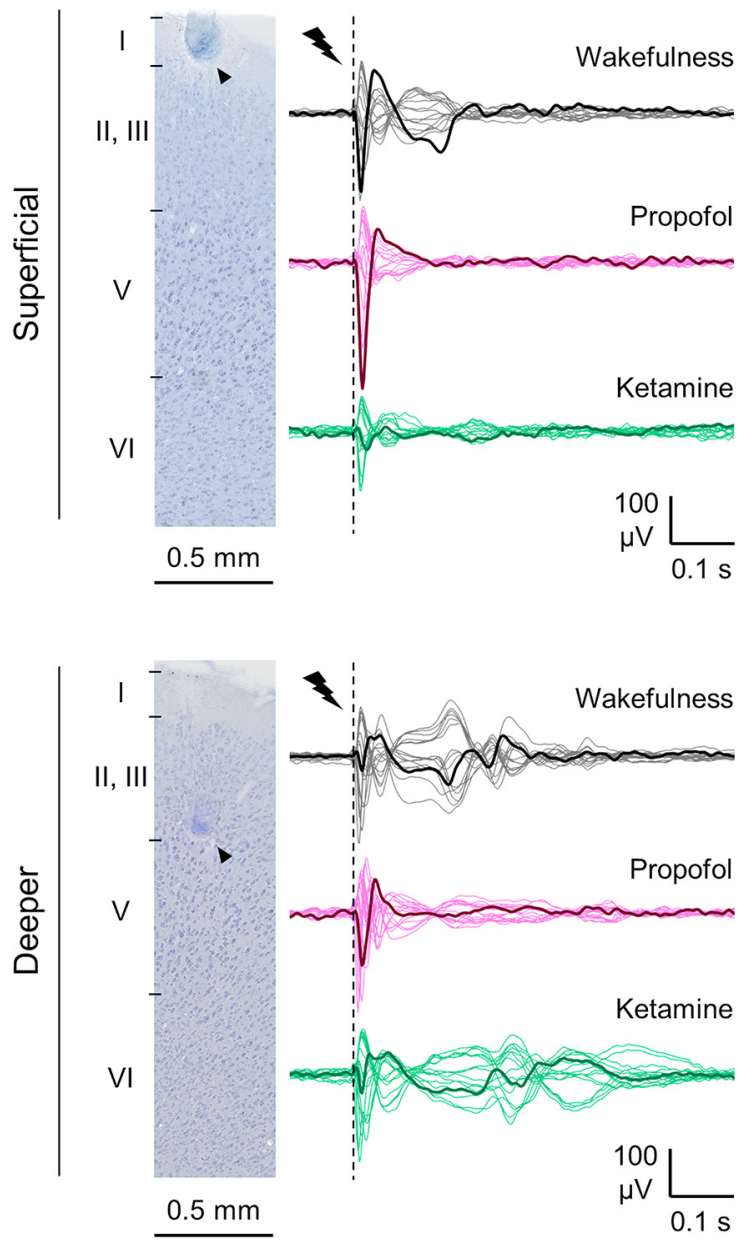

B

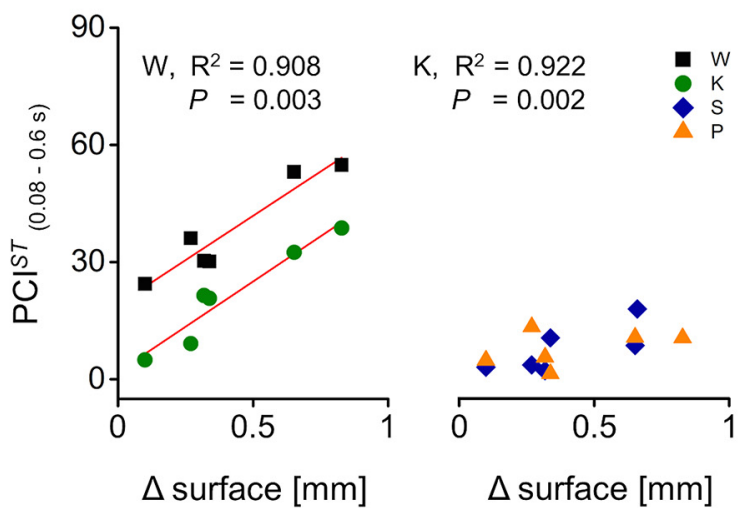

Figure 6. $\mathrm{PCl}^{S T}$ positively correlated with the depth of the stimulation site within the M2 cortex during wakefulness and ketamine anesthesia, but not with propofol or sevoflurane. $\boldsymbol{A}$, left, Coronal cortical sections (Nissl staining) showing the location of the electrode for electrical stimulation in the right M2 cortex from one rat with the tip of the electrode positioned close to the cortical surface (top panel, superficial) and from another animal with the tip of the electrode deeper implanted in the cortex (bottom panel: deeper). Black arrowheads indicate the marks of
By contrast, we did not find any significant correlation between $\mathrm{PCl}^{\mathrm{ST}}{ }_{(0.08-0.6 \mathrm{~s})}$ and stimulation depth during both propofol and sevoflurane anesthesia (linear fit, propofol: $p=0.387$, sevoflurane: $p=0.082$; Fig. $6 A, B$ ). Finally, we repeated the main analysis within only the group of rats that had the stimulating electrodes located within the same cortical Layer II/III (Extended Data Fig. 6-3). Also, within this subgroup of five animals, we found the same relations across conditions as shown for the group of all animals (Extended Data Fig. 6-3). This result indicates that the observed dependency between stimulation depth and $\mathrm{PCl}^{S T}$ did not affect the comparisons between wakefulness and anesthesia of our experiments.

\section{Discussion}

In this study, we established a method for reliable, chronic recording of multichannel, epidural EEG in response to local, cortical, electrical stimulation in rats (Fig. 1; Extended Data Figs. 1-1, 1-2, 1-3). We then successfully used this method to quantify the $\mathrm{PCl}$ (Casali et al., 2013). $\mathrm{PCl}$ is a proposed measure of the brain's capacity to sustain complex dynamics and conscious brain states (Casali et al., 2013) and here, a new variant of this measure $\left(\mathrm{PCl}^{S T}\right)$ has been adopted, since it is more suitable for intracranial electrical stimulation (Comolatti et al., 2019). Both $\mathrm{PCl}$ and $\mathrm{PCl}^{S T}$ were extensively validated on humans as reliable consciousness metrics (Casali et al., 2013; Sarasso et al., 2015; Casarotto et al., 2016; Rosanova et al., 2018; Comolatti et al., 2019), and here we directly applied a $\mathrm{PCl}$ measure for the first time in a non-human animal species in vivo, by using the same parameters adopted for humans (Comolatti et al., 2019), thus allowing direct interspecies comparison and validation.

For measuring $\mathrm{PCl}^{S T}$, we stimulated the M2 cortex because it is a highly integrated area in rodent neocortex (Zingg et al., 2014; Barthas and Kwan, 2017), suitable for triggering

\section{continued}

one pole of the stimulating electrode. Right, Superimposition of mean ERPs from all recording electrodes in response to single pulse stimulation ( $1 \mathrm{~ms}, 50 \mu \mathrm{A}$; dashed line) from the same two rats shown on the left, during wakefulness $(\mathrm{W})$ and propofol $(\mathrm{P})$ and ketamine $(\mathrm{K})$ anesthesia. One averaged ERP from the same channel (S1) is shown in bold to highlight differences across conditions. $\boldsymbol{B}$, Values of $\mathrm{PCl}^{S T}$ (in time range: $0.08-0.6 \mathrm{~s}$ ) from six rats and for all conditions are plotted against the corresponding distances of the stimulating electrode tips from the cortical surface and linearly fitted (coefficient of determination $R^{2}$ and $p$ value are reported if $p<0.05$ ). Strong positive correlations were identified for wakefulness and ketamine conditions with similar slopes (45.35 and 46.30, respectively), but not for propofol and sevoflurane (S) anesthesia. See Extended Data Figure 6-1 for in depth examination of correlation of $\mathrm{PCI}^{S T}$ with stimulus location in wakefulness (examples of ERPs with sevoflurane are also shown). See Extended Data Figure 6-2 for correlations with stimulus location of number of principal components and state transitions, HF power and ITPC drop time, in all conditions. See Extended Data Figure 6-3 for in depth analysis across conditions, with only those rats with confirmed colocalization of stimulating electrodes in Layer II/III. 
widespread and differentiated responses, and it resembles human premotor cortex, often used for probing PCl (Casali et al., 2013; Sarasso et al., 2015). Within M2, we targeted Layer II/III because it contains mainly cell bodies of pyramidal neurons that project to other ipsilateral and contralateral cortical areas (Petreanu et al., 2007; Harris and Shepherd, 2015; Gerfen et al., 2018), and thus seemed suitable for investigating corticocortical integration. We tested complexity during wakefulness and general anesthesia induced by propofol, sevoflurane, and ketamine, similarly to a previous study in humans (Sarasso et al., 2015), thus allowing a direct comparison across species. For each anesthetic drug, the depth of anesthesia was set at the minimal dose that abolished all motor responses to pain stimulation, and spontaneous movements were also not seen. At the same level of behavioral unresponsiveness, propofol and sevoflurane induced a spontaneous EEG activity characterized by high amplitude, LF waves, while ketamine anesthesia yielded sustained, fast, desynchronized EEG activity, resembling the awake state. In agreement with previous studies in humans (Colombo et al., 2019; Lendner et al., 2019), the spectral exponent of the relative periodogram $(20-40 \mathrm{~Hz})$ was reduced by propofol and sevoflurane, but with ketamine it remained similar to wakefulness (Figs. 1, 4; Extended Data Fig. 4-1). During wakefulness, the electrical stimulation produced a long-lasting and complex ERP with a sequence of reproducible voltage inversions in time and across cortical areas. The ERP had a peak power in the 8 - to $25-\mathrm{Hz}$ range, but reached up to $40 \mathrm{~Hz}$ and remained phase-locked for $\sim 0.3 \mathrm{~s}$. Conversely, in propofol anesthesia, the ERP was short-lasting, with few polarity changes, and after a brief HF response, ITPC dropped within $\sim 0.1 \mathrm{~s}$ (Figs. 1, 2). Thus, $\mathrm{PCI}^{S T}$ was reduced from wakefulness to propofol anesthesia in all rats (Fig. 1; Extended Data Fig. 3-2), in agreement with previous results from humans (Sarasso et al., 2015).

Next, we investigated possible mechanisms underlying the reduced complexity. It has been hypothesized that cortical bistability may be important for preventing complex cortical dynamics (Tononi et al., 2016; SanchezVives et al., 2017; Storm et al., 2017). Specifically, when the arousing input from the brainstem and thalamus is reduced or counteracted, such as during NREM sleep, general anesthesia, or deafferentation, cortical circuits tend to become bi-stable and fall into synchronized down-states (neuronal hyperpolarization) following previous activations, or up-states (Steriade et al., 1993, 2001; Sanchez-Vives and McCormick, 2000; Timofeev et al., 2000; Volgushev et al., 2006). At the network level, these changes give rise to slow wave oscillations in the spontaneous EEG observed during NREM sleep and general anesthesia, where ON-periods of HF activity and firing alternate with OFF-periods of HF suppression and neuronal silence (Steriade et al., 1993, 1996, 2001; Mukovski et al., 2007; Vyazovskiy et al., 2009a,b). Neuronal downstates are thought to be generated mainly by activity-dependent $\mathrm{K}^{+}$currents (Timofeev et al., 2001; Compte et al., 2003) and/or synaptic fatigue and inhibition (Esser et al.,
2007; Funk et al., 2017), and it has been suggested that the same mechanisms may be triggered by the initial response to cortical stimulation (Tononi et al., 2016; Sanchez-Vives et al., 2017). The "induced down-state" would then interrupt the deterministic and long-lasting sequence of complex neuronal interactions that are thought to give high PCl (Tononi et al., 2016; Sanchez-Vives et al., 2017). Indirect evidence for this mechanism was recently observed in humans during NREM sleep and UWS, when an OFF period characterized by HF suppression in the EEG was found to follow the initial response to cortical stimulation, and to correlate with the interruption of the phase-locked activation (Pigorini et al., 2015; Rosanova et al., 2018). Similar results were obtained in cortical slices in vitro, with pharmacological reduction of bistability (D'Andola et al., 2018). In agreement with these results, we found that propofol anesthesia induced a profound and widespread HF suppression, after the initial response, $\sim 0.08 \mathrm{~s}$ after the stimulation, in all animals, suggesting the presence of an OFF period (Fig. 2; Extended Data Fig. 2-3).

Previous experiments in rats showed that during NREM sleep, OFF periods of transient and synchronized neuronal silence coincided with spontaneous slow waves in the EEG (Vyazovskiy et al., 2009b). Moreover, it has been shown that electrical, cortical stimulation in rats during NREM sleep evoked slow waves that were highly similar to the spontaneous slow activity (Vyazovskiy et al., 2009a). Coherently, the electrical stimulation during propofol anesthesia induced an ERP characterized by a phase-locked, SC $(<4 \mathrm{~Hz})$, with a higher amplitude that was closely related to the amplitude of the ongoing spontaneous slow oscillation, and which, on average, coincided in time with the end phase of the HF suppression (Extended Data Fig. 2-1).

Importantly, by increasing the stimulus intensity during propofol anesthesia, we were always able to obtain a similar increased cortical excitation as during wakefulness, but the putative OFF period was never abolished. Instead, both the magnitude and duration of the HF suppression increased linearly with the stimulus intensity (Fig. 3; Extended Data Fig. 2-2), suggesting that the OFF period likely reflected an adaptation-like induced down-state. This is in line with the idea that when bistability dominates, a stronger activation leads to a more hyperpolarized state, and is compatible with results from slow waves in cortical slices, where an increased firing rate during the up-state was followed by longer and more hyperpolarized down-state (Sanchez-Vives et al., 2010). The same mechanism might underlie previous findings in rats, showing that $\mathrm{ON}$ periods with higher neuronal firing rate were followed by longer OFF periods with slow waves of higher amplitude, during early NREM sleep (Vyazovskiy et al., 2009b). Consistently with these results, by increasing stimulus intensity, we found a highly significant correlation between the maximal amplitude of the slow ERP component and the magnitude of the HF suppression (Fig. 3). Importantly, also the latency of maximal HF suppression and the time of the ITPC drop were positively correlated (Fig. 3; Extended Data Fig. 3-1), supporting the 
idea that the OFF period can indeed interrupt the deterministic response to the stimulation, as previously observed in UWS and NREM sleep in humans (Pigorini et al., 2015; Rosanova et al., 2018). Interestingly, we found that the ITPC drop was better temporally related to the end phase of the HF suppression rather than to the time of deepest HF suppression (Extended Data Fig. 3-1) as suggested in previous works (Pigorini et al., 2015; Rosanova et al., 2018). This could be explained either by a temporal imprecision of the wavelet convolution, or by different timing of the termination of firing of the involved neurons within the OFF period. However, also the averaged peak latency of the slow ERP component was found to coincide in time with the end phase of the HF suppression period (Extended Data Fig. 2-1), thus suggesting a common physiological mechanism.

With propofol, the HF response was not simply abolished after the OFF period: stimulation-induced HF activations were still detectable later, even if they were sparse in space and time, and not phase-locked, more resembling a modulation of ongoing activity (David et al., 2006). In contrast, during wakefulness, later HF activations were still phase-locked, sustained and widespread, indicating strong deterministic responses (Figs. 2, 4). However, traces of HF suppression were detected also during wakefulness in response to stimulation, but these never interrupted long-lasting, phase-locked activations. Compared with propofol anesthesia, the HF suppression during wakefulness was shallower, briefer and insensitive to variations in stimulus intensity (Extended Data Fig. 2-3), suggesting a different mechanistic origin. A similar effect during wakefulness was also previously observed in humans, from electrodes close to the stimulation site (Borchers et al., 2011; Pigorini et al., 2015), resembling a momentarily increased recruitment of inhibition caused by vigorous electrical stimulation (Borchers et al., 2011; Pigorini et al., 2015). This is also in line with previous experiments involving electrical stimulation of the cortex of awake rats (Vyazovskiy et al., 2013): a first brief activation in response to stimulation was followed by increased firing of putative inhibitory interneurons, which preceded a period of synchronized neuronal silence (Vyazovskiy et al., 2013). However, in the same experiments, the OFF period was more long-lasting and synchronous after longer periods of continuous wakefulness, thus showing a modulatory effect of sleep pressure on neuronal responsiveness (Vyazovskiy et al., 2013).

It therefore seems important in the future to compare EEG signatures of bistability and $\mathrm{PCl}^{S T}$ also between more graded brain states or sub-states within wakefulness and NREM sleep. In light of our and previous results (Vyazovskiy et al., 2013), one would expect that the tendency of cortical circuits to fall into an OFF period after a brief activation should increase, and $\mathrm{PCI}^{S T}$ should decrease, as a function of the time spent in wakefulness, with the maximal neuronal bistability and minimal $\mathrm{PCI}^{\mathrm{ST}}$ during the early stage of NREM sleep (Vyazovskiy et al., 2009a,b; Pigorini et al., 2015; Casali et al., 2013). This would be consistent with the local sleep events, with localized OFF periods and EEG slow waves, that were found to spontaneously occur in rats during long periods of sleep deprivation (Vyazovskiy et al., 2011). Similarly, one may expect that a gradually reduced level of general anesthesia will reduce the magnitude and duration of the evoked OFF period, with a gradual restoration of $\mathrm{PCl}^{\mathrm{ST}}$. Indeed, Mégevand et al. (2008) found that lighter compared with deeper anesthesia induced a higher number of components in the EEG response to sensory stimulation in mice, with an increased propagation of electrical activation, and recently, a variant of perturbational complexity adapted from an in vitro study was found to increase with reduced anesthesia in mice (Dasilva et al., 2021). Also, within the same level of sleep pressure during wakefulness, neuronal responsiveness and cortical complexity might change substantially depending on the state of activity and arousal. Indeed, it was shown in mice that moderate arousal or alert wakefulness was associated with a stable, hyperpolarized membrane potential in cortical neurons and a better behavioral performance, compared with quiet and active wakefulness (McGinley et al., 2015). Thus, a careful control of these parameters, in association with $\mathrm{PCI}^{S T}$, may provide important insights into the neuronal basis for integration of information in cortical circuits.

It is likely that a cortical network that can sustain the propagation of long-lasting, complex sequences of neuronal activations has a high and diversified level of connectivity (Massimini et al., 2009). To test this idea, we examined the functional connectivity following the stimulation within the frequency range that corresponded to the more long-lasting phase-locked response $(\theta-\alpha, 5-14 \mathrm{~Hz})$, which has also been implicated in long-range interactions across brain regions (Lisman and Jensen, 2013; Hyafil et al., 2015). Available evidence from mice (Tamura et al., 2017) and monkeys (Liebe et al., 2012) support the role of $\theta-\alpha$ oscillations in organizing HF activity and coupling occipital-frontal regions during wakefulness and cognitive tasks. Thus, the $\theta$ - $\alpha$ range seemed well suited for testing the long-range functional network connectivity that is particularly relevant for $\mathrm{PCl}$. We used ISPC as a measure of the consistency of phase-based connectivity across trials, to capture the spatial representation of the deterministic sequence of neuronal events that generates the complexity of the response in time. Consistently, we found a strong positive correlation between the derived $\mathrm{CD}$ and $\mathrm{PCl}^{S T}$ (Fig. 5). Indeed, the response during wakefulness showed a high degree of connectivity with a peak in occipital areas, highlighting a certain diversity in the evoked neuronal activations. In contrast, propofol anesthesia reduced cortical connectivity, and the spatial distribution of the remaining functional connections became more uniform (Fig. 5; Juel et al., 2018), resembling a reduction of both integration and differentiation of the neuronal response. Even if we cannot exclude some persisting information flow between distant neurons, which can be even enhanced during NREM sleep in rats after a behavioral task (Olcese et al., 2018), our finding fits with the known effect of propofol in suppressing long-range spontaneous network interactions (Lewis et al., 2012). Indeed, we found a reduced connectivity for each of the frequency bands examined (from 1 to $40 \mathrm{~Hz}$; Extended 
Data Fig. 5-2). Our results are also consistent with the reduced variability in the complexity of evoked responses to stimulations at different cortical depths, observed here (Fig. 6). $\mathrm{PCl}^{S T}$ was indeed found to linearly increase with the cortical depth of stimulation during wakefulness, but not with propofol, within the tested range across rats (from Layer I to upper part of Layer V, with most positions within Layer II/III; Fig. 6; Extended Data Fig. 6-1). This linear relation has to our knowledge not been reported before, presumably because most $\mathrm{PCl}$ experiments were done in humans with TMS, and indicates a conserved differentiation during wakefulness that fades with propofol anesthesia.

We next used the same approach during sevoflurane anesthesia. Sevoflurane and propofol are known to share some molecular mechanisms, such as enhancement of $\mathrm{GABA}_{\mathrm{A}}$ receptors (Bieda and Maclver, 2004; Sebel et al., 2006) and inhibition of voltage-gated $\mathrm{Na}^{+}$channels (Ouyang et al., 2003, 2009), but there are also important differences. Thus, sevoflurane opens TASK two-pore-domain (2P) $\mathrm{K}^{+}$channels, unlike propofol (Putzke et al., 2007), and differently affects sensory processing (Arena et al., 2017). Nevertheless, sevoflurane produced effects that resembled those with propofol. Soon after the initial response to stimulation, a period of deep HF suppression occurred, preceding an early drop of the phase-locked response. Later on, only sparse and not phase-locked HF activations were detected and both functional connectivity and diversity were reduced compared with wakefulness, leading to low $\mathrm{PCl}^{S T}$ (Extended Data Figs. 4-1, 5-1, 5-2; Figs. 5, 6). Taken together, these results support the hypothesis that the down-state may serve as a general mechanism for interrupting complex cortical dynamics. This mechanism seems indeed to be shared across anesthetic drugs, animal species, and brain states, including NREM sleep (Pigorini et al., 2015) and UWS (Rosanova et al., 2018) in humans.

Nevertheless, our results with ketamine may seem to challenge this idea. Ketamine anesthesia is known to produce a state of behavioral unresponsiveness with "dream-like," vivid experiences (Collier, 1972). A previous study in humans found no significant difference in PCl between wakefulness and ketamine anesthesia (Sarasso et al., 2015), but signs of bistability were not examined in this condition (Sarasso et al., 2015). Here, we found that ERPs during ketamine showed mixed features: the initial brief response was rapidly followed ( $\sim 0.08 \mathrm{~s}$ later) by an OFF period, resembling propofol anesthesia, but this did not prevent long-lasting deterministic activations in nearly half the animals, and the duration of the resulting phaselocked response was close to that of wakefulness (Fig. 4). After the OFF period, a later increase in HF activity was highly frequent, but neither consistently phase-locked and deterministic, like in wakefulness, nor consistently not phase-locked, like with propofol and sevoflurane (Fig. 4). During ketamine anesthesia, the time course of $\mathrm{PCI}^{S T}$ revealed similarities to wakefulness, but resulting in an overall reduction of complexity. However, $\mathrm{PCl}^{S T}$ from the onset of the OFF period was significantly higher than in propofol/sevoflurane anesthesia, thus indicating an intermediate level of perturbational complexity with ketamine, regardless of the similar state of behavioral unresponsiveness (Fig. 4). Consistently, the functional connectivity across cortical regions was reduced compared with wakefulness during the OFF period, but recovered soon afterward in the $\theta$ frequency range $(5-7 \mathrm{~Hz}$; Extended Data Fig. 5-2) and a wakefulness-like pattern of $C D$ across space was maintained, suggesting conserved integration and diversity (Fig. 5). Another important sign of a conserved differentiation during ketamine anesthesia was the positive linear relation between $\mathrm{PCl}^{S T}$ and the depth of the site of stimulation within M2, which was also observed in wakefulness, with similar slopes (Fig. 6). The mixed results with ketamine may suggest some variability or unstable effects of this drug. In support of this idea, a recent study in humans found that the EEG activity spontaneously fluctuated between states of low and high complexity during ketamine anesthesia, until awakening ( $\mathrm{Li}$ and Mashour, 2019). Certainly, since our experimental design was adopted to reproduce experiments in humans, we could only indirectly infer signs of down-states by detecting OFF periods of HF suppression in EEG. Indeed, HF suppression is a more direct indication of reduced neuronal activation relative to ongoing activity, and does not necessarily indicate neuronal hyperpolarization, which can be observed only by intracellular recordings. For example, a coordinated reduction in presynaptic release might also produce an OFF period in the EEG, if compared with the enhanced spontaneous activity that is typically induced by ketamine (Brown et al., 2010; Ferro et al., 2017), without implying hyperpolarization. Although our data cannot yet resolve this issue, they illustrate the need for intracellular recordings to determine the role of bistability in the interruption of complex dynamics. Moreover, the novel correlation that we found between $\mathrm{PCl}^{S T}$ and the cortical depth of stimulation represents a promising starting point for studying the roles of specific cortical layers and micro circuitries in sustaining cortical complexity.

In conclusion, we demonstrated that rodent cortical circuits can respond to focal stimulation with long-lasting sequences of deterministic, complex interactions during wakefulness, which are disrupted during general anesthesia, consistently with previous results in humans (Sarasso et al., 2015). In agreement with previous studies (Pigorini et al., 2015; D'Andola et al., 2018; Rosanova et al., 2018), we provided indirect evidence for a connection between cortical bistability, interruption of deterministic responses, and disruption of cortical connectivity and complexity. Our results improve our understanding of the cortical dynamics that in humans have been associated with consciousness, and our method opens a range of future possibilities for more detailed, mechanistic investigations of brain states and their transitions.

\section{References}

Arena A, Lamanna J, Gemma M, Ripamonti M, Ravasio G, Zimarino V, De Vitis A, Beretta L, Malgaroli A (2017) Linear transformation of the encoding mechanism for light intensity underlies the paradoxical enhancement of cortical visual responses by sevoflurane. $J$ Physiol 595:321-339. 
Arena A, Comolatti R, Casali AG, Storm JF (2018) Cortical complexity and cause-effect power are reduced by general anesthesia compared to wakefulness in rats. Program No. 125.26 2018 Neuroscience Meeting Planner. San Diego: Society for Neuroscience.

Arena A, Comolatti R, Thon S, Casali AG, Storm JF (2020) General anaesthesia disrupts complex cortical dynamics in response to intracranial electrical stimulation in rats. bioRxiv 2020.02.25.964056.

Barthas F, Kwan AC (2017) Secondary motor cortex: where "sensory" meets "motor" in the rodent frontal cortex. Trends Neurosci 40:181-193.

Benito J, Aguado D, Abreu MB, García-Fernández J, Gómez de Segura IA (2010) Remifentanil and cyclooxygenase inhibitors interactions in the minimum alveolar concentration of sevoflurane in the rat. Br J Anaesth 105:810-817.

Bieda MC, Maclver MB (2004) Major role for tonic GABAA conductances in anesthetic suppression of intrinsic neuronal excitability. J Neurophysiol 92:1658-1667.

Borchers S, Himmelbach M, Logothetis N, Karnath HO (2011) Direct electrical stimulation of human cortex - the gold standard for mapping brain functions? Nat Rev Neurosci 13:63-70.

Brammer A, West CD, Allen SL (1993) A comparison of propofol with other injectable anaesthetics in a rat model for measuring cardiovascular parameters. Lab Anim 27:250-257.

Brecht M, Krauss A, Muhammad S, Sinai-Esfahani L, Bellanca S, Margrie TW (2004) Organization of rat vibrissa motor cortex and adjacent areas according to cytoarchitectonics, microstimulation, and intracellular stimulation of identified cells. J Comp Neurol 479:360-373.

Brown EN, Lydic R, Schiff ND (2010) General anesthesia, sleep, and coma. N Engl J Med 363:2638-2650.

Casali AG, Gosseries O, Rosanova M, Boly M, Sarasso S, Casali KR, Casarotto S, Bruno M-A, Laureys S, Tononi G, Massimini M (2013) A theoretically based index of consciousness independent of sensory processing and behavior. Sci Transl Med 5:1-10.

Casarotto S, Comanducci A, Rosanova M, Sarasso S, Fecchio M, Napolitani M, Pigorini AG, Casali A, Trimarchi PD, Boly M, Gosseries O, Bodart O, Curto F, Landi C, Mariotti M, Devalle G, Laureys S, Tononi G, Massimini M (2016) Stratification of unresponsive patients by an independently validated index of brain complexity. Ann Neurol 80:718-729.

Cohen MX (2014) Analyzing neural time series data: theory and practice, Ed 1. Cambridge: The MIT Press.

Collier BB (1972) Ketamine and the conscious mind. Anaesthesia 27:120-134.

Colombo MA, Napolitani M, Boly M, Gosseries O, Casarotto S, Rosanova M, Brichant JF, Boveroux P, Rex S, Laureys S, Massimini M, Chieregato A, Sarasso S (2019) The spectral exponent of the resting EEG indexes the presence of consciousness during unresponsiveness induced by propofol, xenon, and ketamine. Neuroimage 189:631-644.

Comolatti R, Pigorini A, Casarotto S, Fecchio M, Faria G, Sarasso S, Rosanova M, Gosseries O, Boly M, Bodart O, Ledoux D, Brichant JF, Nobili L, Laureys S, Tononi G, Massimini M, Casali AG (2019) A fast and general method to empirically estimate the complexity of brain responses to transcranial and intracranial stimulations. Brain Stimul 12:1280-1289.

Compte A, Sanchez-Vives MV, McCormick DA, Wang X-J (2003) Cellular and network mechanisms of slow oscillatory activity $(<1$ $\mathrm{Hz}$ ) and wave propagations in a cortical network model. J Neurophysiol 89:2707-2725.

D'Andola M, Rebollo B, Casali AG, Weinert JF, Pigorini A, Villa R, Massimini M, Sanchez-Vives MV (2018) Bistability, causality, and complexity in cortical networks: an in vitro perturbational study. Cereb Cortex 28:2233-2242.

Dasilva M, Camassa A, Navarro-Guzman A, Pazienti A, PerezMendez L, Zamora-López G, Mattia M, Sanchez-Vives MV (2021) Modulation of cortical slow oscillations and complexity across anesthesia levels. Neuroimage 224:117415.
David O, Kilner JM, Friston KJ (2006) Mechanisms of evoked and induced responses in MEG/EEG. Neuroimage 31:1580-1591.

De Farias Rocha FA, Gomes BD, De Lima Silveira LC, Martins SL, Aguiar RG, De Souza JM, Ventura DF (2016) Spectral sensitivity measured with electroretinogram using a constant response method. PLoS One 11:e0147318.

Dehaene S (2014) Consciousness and the brain: deciphering how the brain codes our thoughts. New York: Penguin.

Demertzi A, Tagliazucchi E, Dehaene S, Deco G, Barttfeld P, Raimondo F, Martial C, Fernández-Espejo D, Rohaut B, Voss HU, Schiff ND, Owen AM, Laureys S, Naccache L, Sitt JD (2019) Human consciousness is supported by dynamic complex patterns of brain signal coordination. Sci Adv 5:eaat7603.

Esser SK, Hill SL, Tononi G (2007) Sleep homeostasis and cortical synchronization: I. Modeling the effects of synaptic strength on sleep slow waves. Sleep 30:1617-1630.

Ferro M, Lamanna J, Ripamonti M, Racchetti G, Arena A, Spadini S, Montesano G, Cortese R, Zimarino V, Malgaroli A (2017) Functional mapping of brain synapses by the enriching activitymarker SynaptoZip. Nat Commun 8:1229.

Funk CM, Peelman K, Bellesi M, Marshall W, Cirelli C, Tononi G (2017) Role of somatostatin-positive cortical interneurons in the generation of sleep slow waves. J Neurosci 37:9132-9148.

Gerfen CR, Economo MN, Chandrashekar J (2018) Long distance projections of cortical pyramidal neurons. J Neurosci Res 96:1467-1475.

Harris KD, Shepherd GMG (2015) The neocortical circuit: themes and variations. Nat Neurosci 18:170-181.

Ho J, Tumkaya T, Aryal S, Choi H, Claridge-Chang A (2019) Moving beyond $p$ values: data analysis with estimation graphics. Nat Methods 16:565-566.

Hyafil A, Giraud AL, Fontolan L, Gutkin B (2015) Neural cross-frequency coupling: connecting architectures, mechanisms, and functions. Trends Neurosci 38:725-740.

Idvall J, Aronsen KF, Stenberg P (1980) Tissue perfusion and distribution of cardiac output during ketamine anesthesia in normovolemic rats. Acta Anaesthesiol Scand 24:257-263.

Jacobs GH, Fenwick JA, Williams GA (2001) Cone based vision of rats for ultraviolet and visible lights. J Exp Biol 204:2439-2446.

Juel BE, Romundstad L, Kolstad F, Storm JF, Larsson PG (2018) Distinguishing anesthetized from awake state in patients: a new approach using one second segments of raw EEG. Front Hum Neurosci 12:40.

Koch C, Massimini M, Boly M, Tononi G (2016) Neural correlates of consciousness: progress and problems. Nat Rev Neurosci 17:307-321.

Lendner JD, Helfrich RF, Mander BA, Romundstad L, Lin JJ, Walker MP, Larsson PG, Knight RT (2020) An electrophysiological marker of arousal level in humans. eLife 9:e55092.

Lewis LD, Weiner VS, Mukamel EA, Donoghue JA, Eskandar EN, Madsen JR, Anderson WS, Hochberg LR, Cash SS, Brown EN, Purdon PL (2012) Rapid fragmentation of neuronal networks at the onset of propofol-induced unconsciousness. Proc Natl Acad Sci USA 109:E3377-E3386.

Li D, Mashour GA (2019) Cortical dynamics during psychedelic and anesthetized states induced by ketamine. Neuroimage 196:32-40.

Liebe S, Hoerzer GM, Logothetis NK, Rainer G (2012) Theta coupling between V4 and prefrontal cortex predicts visual short-term memory performance. Nat Neurosci 15:456-462.

Lisman JE, Jensen O (2013) The theta-gamma neural code. Neuron 77:1002-1016.

Lopes G, Bonacchi N, Frazão J, Neto JP, Atallah BV, Soares S, Moreira L, Matias S, Itskov PM, Correia PA, Medina RE, Calcaterra L, Dreosti E, Paton JJ, Kampff AR (2015) Bonsai: an event-based framework for processing and controlling data streams. Front Neuroinform 9:7-14.

Marwan N, Carmen RM, Thiel M, Kurths J (2007) Recurrence plots for the analysis of complex systems. Phys Rep 438:237-329. 
Massimini M, Boly M, Casali A, Rosanova M, Tononi G (2009) A perturbational approach for evaluating the brain's capacity for consciousness. Prog Brain Res 177:201-214.

McGinley MJ, David SV, McCormick DA (2015) Cortical membrane potential signature of optimal states for sensory signal detection. Neuron 87:179-192.

Mégevand P, Quairiaux C, Lascano AM, Kiss JZ, Michel CM (2008) A mouse model for studying large-scale neuronal networks using EEG mapping techniques. Neuroimage 42:591-602.

Mukovski M, Chauvette S, Timofeev I, Volgushev M (2007) Detection of active and silent states in neocortical neurons from the field potential signal during slow-wave sleep. Cereb Cortex 17:400-414.

Olcese U, Bos JJ, Vinck M, Pennartz CMA (2018) Functional determinants of enhanced and depressed interareal information flow in nonrapid eye movement sleep between neuronal ensembles in rat cortex and hippocampus. Biochem Pharmacol 24:1639-1618.

Ouyang W, Wang G, Hemmings HC (2003) Isoflurane and propofol inhibit voltage-gated sodium channels in isolated rat neurohypophysial nerve terminals. Mol Pharmacol 64:373-381.

Ouyang W, Herold KF, Hemmings HC (2009) Comparative effects of halogenated inhaled anesthetics on voltage-gated $\mathrm{Na}+$ channel function. Anesthesiology 110:582-590.

Petreanu L, Huber D, Sobczyk A, Svoboda K (2007) Channelrhodopsin2-assisted circuit mapping of long-range callosal projections. Nat Neurosci 10:663-668.

Pigorini A, Sarasso S, Proserpio P, Szymanski C, Arnulfo G, Casarotto S, Fecchio M, Rosanova M, Mariotti M, Lo Russo G, Palva JM, Nobili L, Massimini M (2015) Bistability breaks-off deterministic responses to intracortical stimulation during non-REM sleep. Neuroimage 112:105-113.

Putzke C, Hanley PJ, Schlichthörl G, Preisig-Müller R, Rinné S, Anetseder M, Eckenhoff R, Berkowitz C, Vassiliou T, Wulf $H$, Eberhart L (2007) Differential effects of volatile and intravenous anesthetics on the activity of human TASK-1. Am J Physiol Cell Physiol 293:C1319-C1326.

Rosanova M, Fecchio M, Casarotto S, Sarasso S, Casali AG, Pigorini A, Comanducci A, Seregni F, Devalle G, Citerio G, Bodart O, Boly M, Gosseries O, Laureys S, Massimini M (2018) Sleep-like cortical OFF-periods disrupt causality and complexity in the brain of unresponsive wakefulness syndrome patients. Nat Commun 9:4427.

Sanchez-Vives MV, McCormick DA (2000) Cellular and network mechanisms of rhythmic recurrent activity in neocortex. Nat Neurosci 3:1027-1034.

Sanchez-Vives MV, Mattia M, Compte A, Perez-Zabalza M, Winograd M, Descalzo VF, Reig R (2010) Inhibitory modulation of cortical up states. J Neurophysiol 104:1314-1324.

Sanchez-Vives MV, Massimini M, Mattia M (2017) Shaping the default activity pattern of the cortical network. Neuron 94:993-1001.

Sarasso S, Boly M, Napolitani M, Gosseries O, Charland-Verville V, Casarotto S, Rosanova M, G, Casali A, Brichant J-F, Boveroux P, Rex S, Tononi G, Laureys S, Massimini M (2015) Consciousness and complexity during unresponsiveness induced by propofol, xenon, and ketamine. Curr Biol 25:3099-3105.
Schartner M, Seth A, Noirhomme Q, Boly M, Bruno MA, Laureys S, Barrett A (2015) Complexity of multi-dimensional spontaneous EEG decreases during propofol induced general anaesthesia. PLoS One 10:e0133532.

Sebel LE, Richardson JE, Singh SP, Bell SV, Jenkins A (2006) Additive effects of sevoflurane and propofol on gamma-aminobutyric acid receptor function. Anesthesiology 104:1176-1183.

Siegle JH, López AC, Patel YA, Abramov K, Ohayon S, Voigts J (2017) Open Ephys: an open-source, plugin-based platform for multichannel electrophysiology. J Neural Eng 14:045003.

Steriade M, Nuñez A, Amzica F (1993) A novel slow $(<1 \mathrm{~Hz}$ ) oscillation of neocortical neurons in vivo: depolarizing and hyperpolarizing components. J Neurosci 13:3252-3265.

Steriade M, Contreras D, Amzica F, Timofeev I (1996) Synchronization of fast $(30-40 \mathrm{~Hz})$ spontaneous oscillations in intrathalamic and thalamocortical networks. J Neurosci 16:2788-2808.

Steriade M, Timofeev I, Grenier F (2001) Natural waking and sleep states: a view from inside neocortical neurons. J Neurophysiol 85:1969-1985.

Storm JF, Boly M, Casali AG, Massimini M, Olcese U, Pennartz CMA, Wilke M (2017) Consciousness regained: disentangling mechanisms, brain systems, and behavioral responses. J Neurosci 37:10882-10893.

Tamura M, Spellman TJ, Rosen AM, Gogos JA, Gordon JA (2017) Hippocampal-prefrontal theta-gamma coupling during performance of a spatial working memory task. Nat Commun 8:2182.

Timofeev I, Grenier F, Bazhenov M, Sejnowski TJ, Steriade M (2000) Origin of slow cortical oscillations in deafferented cortical slab. Cereb Cortex 10:1185-1199.

Timofeev I, Grenier F, Steriade M (2001) Disfacilitation and active inhibition in the neocortex during the natural sleep-wake cycle: an intracellular study. Proc Natl Acad Sci USA 98:1924-1929.

Tononi G, Boly M, Massimini M, Koch C (2016) Integrated information theory: from consciousness to its physical substrate. Nat Rev Neurosci 17:450-461.

Volgushev M, Chauvette S, Mukovski M, Timofeev I (2006) Precise long-range synchronization of activity and silence in neocortical neurons during slow-wave oscillations. J Neurosci 26:5665-5672.

Vyazovskiy VV, Faraguna U, Cirelli C, Tononi G (2009a) Triggering slow waves during NREM sleep in the rat by intracortical electrical stimulation: effects of sleep/wake history and background activity. J Neurophysiol 101:1921-1931.

Vyazovskiy VV, Olcese U, Lazimy YM, Faraguna U, Esser SK, Williams JC, Cirelli C, Tononi G (2009b) Cortical firing and sleep homeostasis. Neuron 63:865-878.

Vyazovskiy VV, Olcese U, Hanlon EC, Nir Y, Cirelli C, Tononi G (2011) Local sleep in awake rats. Nature 472:443-447.

Vyazovskiy VV, Olcese U, Cirelli C, Tononi G (2013) Prolonged wakefulness alters neuronal responsiveness to local electrical stimulation of the neocortex in awake rats. J Sleep Res 22:239-250.

Zingg B, Hintiryan H, Gou L, Song MY, Bay M, Bienkowski MS, Foster NN, Yamashita S, Bowman I, Toga AW, Dong HW (2014) Neural networks of the mouse neocortex. Cell 156:1096-1111. 